\title{
MODAL LOGICS OF TOPOLOGICAL RELATIONS
}

\author{
CARSTEN LUTZ $^{a}$ AND FRANK WOLTER ${ }^{b}$ \\ ${ }^{a}$ Institute of Theoretical Computer Science TU Dresden, Germany \\ e-mail address: lutz@tcs.inf.tu-dresden.de \\ ${ }^{b}$ Department of Computer Science, University of Liverpool, United Kingdom \\ e-mail address: frank@csc.liv.ac.uk
}

\begin{abstract}
Logical formalisms for reasoning about relations between spatial regions play a fundamental role in geographical information systems, spatial and constraint databases, and spatial reasoning in AI. In analogy with Halpern and Shoham's modal logic of time intervals based on the Allen relations, we introduce a family of modal logics equipped with eight modal operators that are interpreted by the Egenhofer-Franzosa (or RCC8) relations between regions in topological spaces such as the real plane. We investigate the expressive power and computational complexity of logics obtained in this way. It turns out that our modal logics have the same expressive power as the two-variable fragment of first-order logic, but are exponentially less succinct. The complexity ranges from (undecidable and) recursively enumerable to $\Pi_{1}^{1}$-hard, where the recursively enumerable logics are obtained by considering substructures of structures induced by topological spaces. As our undecidability results also capture logics based on the real line, they improve upon undecidability results for interval temporal logics by Halpern and Shoham. We also analyze modal logics based on the five RCC5 relations, with similar results regarding the expressive power, but weaker results regarding the complexity.
\end{abstract}

\section{INTRODUCTION}

Reasoning about topological relations between regions in space is recognized as one of the most important and challenging research areas within spatial reasoning in artificial intelligence (AI) and philosophy, spatial and constraint databases, and geographical information systems (GISs). Research in this area can be classified according to the logical apparatus employed:

- First-order theories of topological relations between regions, as studied in AI and philosophy Cla85, RCC92, PS98, CH01, spatial databases PSV99, SS01] and from an algebraic viewpoint in DWM01, Ste00, DW05;

- Purely existential theories formulated as constraint satisfaction systems over jointly exhaustive and mutually disjoint sets of topological relations between regions Ege94, RN99, GPP95, SS01, RCC92, Ben94, CH01,

2000 ACM Subject Classification: F4.1, H2.8, I2.4.

Key words and phrases: Spatial reasoning, topology, modal logic, RCC8, expressive completeness, decidability, axiomatizability. 
- Modal logics of space with operators interpreted by the closure and interior operator of the underlying topological space and propositions interpreted as subsets of the topological space, see e.g., KT44, Ben96, AvB02, Nut99, PH02,

A similar classification can be made for temporal reasoning: we have general first-order theories [All84, temporal constraint systems All83, VKV90, NB95] and modal temporal logics like Prior's tense logics, LTL, and CTL GHR94, Eme90. Surprisingly, one of the most natural approaches to temporal reasoning has not yet found a fully developed analogue on the spatial reasoning research agenda: Halpern and Shoham's modal logic of intervals HS91, in which propositions are evaluated at intervals (rather than time points), and where reference to other intervals is enabled by modal operators interpreted by Allen's 13 relations between intervals, see also vB83, Gal87. Despite its bad computational behavior (undecidable, usually not even r.e.), this framework proved rather fruitful and influential in temporal reasoning, see e.g. Ven90, Ven92, AF98, Ras99, Lod00, Lut03.

In this paper, we consider modal logics in which propositions are evaluated at the regions of topological spaces, and reference to other regions is enabled by modal operators interpreted as topological relations. For defining such logics, the two most important decisions to be made are choosing an appropriate set of relations and identifying a suitable notion of a "region" in a topological space.

Regarding the relations, in the initially mentioned research areas there appears to be consensus that the eight Egenhofer-Franzosa (or RCC8) relations, which have been independently introduced in [RCC92] and EF91, and their coarser relative RCC5 consisting of only five relations, are the most fundamental sets of relations between regions of topological spaces - both from a theoretical and a practical viewpoint, see e.g. PSV99, Ege94, RN99, SS01, RCC92]. Therefore, in the current paper we concentrate on these two sets of relations. We should note that modal logics based on the Egenhofer-Franzosa relations have been suggested in an early paper by Cohn Coh93] and further considered in Wes01. However, it proved difficult to analyze the expressive power and computational behavior of such logics: despite several efforts, to the best of our knowledge no results have been obtained so far.

Concerning the regions of a topological space, we adopt a rather relaxed view: we generally assume that regions are non-empty regular closed subsets of a topological space, but we do not require that every such subset is a region. This view allows us to consider logical structures, henceforth called region structures, that are based on various kinds of regions. Among others, we consider the following options:

- Region structures in which the set of regions is exactly the set of non-empty regular closed subsets of a topological space.

- In the Euclidean space $\mathbb{R}^{n}$, region structures where regions are identified with all nonempty convex regular closed sets, or with all hyper-rectangles.

- Substructures of the above region structures: for example, we may admit region structures in which only some, but not all hyper-rectangles of $\mathbb{R}^{n}$ are regions. To distinguish this case from the former two, we call region structures in which all regions of a particular kind are present full region structures.

- Finite substructures of the above region structures.

The rationale behind the latter two choices of structures is that, for certain applications, it is sufficient to require the presence of only those regions in region structures that are 
inhabited by spatial objects. If it is known that there are only finitely many such objects, but their exact number is unknown, then finite substructures are the appropriate choice.

The main purpose of this paper is to introduce modal logics of topological relations in a systematic way, to perform an investigation of their expressiveness and relationships, and to analyze their computational behavior. Regarding expressiveness, our main result concerns the relationship to first-order theories of topological relations. The expressive power of our modal logics is incomparable with that standard theories of this kind since modal logics offer an infinite supply of propositional variables corresponding to unary predicates of first-order logic. In contrast, standard first-order theories of topological relations offer only eight binary predicates interpreted as topological relations, and no unary predicates RCC92, PS98, PSV99, SS01. Therefore, we consider the extension of first-order theories of topological relations with an infinite number of "free" unary predicates. Then, we can show that our logics based on the Egenhofer-Franzosa or RCC5 relations has exactly the same expressive power as the two-variable fragment of first-order logic on the same set of relations (indeed, this holds for any mutually disjoint and jointly exhaustive set of topological relations). We also show that first-order logic is exponentially more succinct. We argue that the availability of unary predicates is essential for a wide range of application areas: in contrast to describing only purely topological properties of regions, it allows one to also capture other properties such as being a country (in a GIS), a ball (for a soccer-playing robot), or a protected area (in a spatial database). In our modal logics, we can thus formulate constraints based on non-spatial properties such as "there are no two overlapping regions that are both countries" and "every river is connected to an ocean or a lake".

The main results of this paper concern the computational behavior of modal logics of topological relations. We prove a very general undecidability result that captures all modal logics of the RCC8 relations that are determined by a class of region structures whose regions are (not necesserily all) non-empty regular closed sets, and that contains at least one infinite structure. It is interesting to note that this result also covers logics that are determined by substructures of region structures. In particular, it captures the substructures of the real line where regions are intervals, and thus improves upon undecidability results for interval temporal logics by Halpern and Shoham that do not capture substructures of interval structures HS91. Using a variation of the proof of our central theorem, we can even show that logics based on finite substructures of region structures are undecidable. Although our results show that moving from full region structures to substructures does not help to regain decidability, there is an improvement in computational complexity: we show that most logics of RCC8 relations based on full region structures are $\Pi_{1}^{1}$-hard and thus not recursively enumerable. In contrast, we also prove that many logics determined by substructures are recursively enumerable. Finally, we establish the undecidability of a number of modal logics based on the RCC5 relations. The result is less general and, for example, does not cover the substructure case. Recursive enumerability of RCC5-based logics is left as an open problem.

This paper is organized as follows: in Section 2] we introduce region structures as the semantical basis for modal logics of topological relations. The modal language is introduced in Section 3. In this section, we also compare its expressiveness to that of first-order logic. Additionally, we show that our modal logics are strictly more expressive than topological constraint satisfaction problems. In Section [4 we introduce a number of natural modal logics based on the Egenhofer-Franzosa relations that are induced by different notions of regions, and briefly analyze their relationship. In Section [5 we then prove the central 
undecidability result capturing basically all interesting modal logics of RCC8 relations determined by sets of region structures containing at least one infinite structure. For logics of full region structures, this is strengthened to a $\Pi_{1}^{1}$-hardness proof in Section [6 We also prove recursive enumerability of many modal logics based on substructures of region structures. In Section 7 we prove undecidability of logics determined by classes of finite region structures. Finally, in Section 8 we consider modal logics based on the RCC5 relations.

\section{Structures}

The purpose of the logics considered in this paper is to reason about regions in topological spaces. In this section, we show how a topological space together with an appropriate definition of "region" induces a logical structure, and establish some basic properties of the structures obtained in this way.

Recall that a topological space is a pair $\mathfrak{T}=(U, \mathbb{I})$, where $U$ is a set and $\mathbb{I}$ is an interior operator on $U$, i.e., for all $s, t \subseteq U$, we have

$$
\begin{array}{rlrl}
\mathbb{I}(U) & =U & \mathbb{I}(s) & \subseteq s \\
\mathbb{I}(s) \cap \mathbb{I}(t) & =\mathbb{I}(s \cap t) & \mathbb{I}(s) & =\mathbb{I}(s) .
\end{array}
$$

The closure $\mathbb{C}(s)$ of $s$ is $\mathbb{C}(s)=U-\mathbb{I}(U-s)$. Of particular interest for spatial reasoning are $n$-dimensional Euclidean spaces $\mathbb{R}^{n}$ based on Cartesian products of the real line with the standard topology induced by the Euclidean metric. Depending on the application domain, different definitions of regions in topological spaces have been introduced. Almost all of them have in common that the regions of a topological space $\mathfrak{T}=(U, \mathbb{I})$ are identified with some set of non-empty, regular closed subsets of $U$, where a subset $s \subseteq U$ is called regular closed if $\mathbb{C I}(s)=s .{ }^{1}$ Some popular choices for topological spaces and regions are the following:

- the set $\mathfrak{T}_{\text {reg }}$ of all non-empty regular closed subsets of some topological space $\mathfrak{T}$, in particular the topological spaces $\mathbb{R}^{n}$ for some $n \geq 1$;

- the set $\mathbb{R}_{\text {conv }}^{n}$ of non-empty convex regular closed subsets of $\mathbb{R}^{n}$, for some $n \geq 1$;

- the set $\mathbb{R}_{\text {rect }}^{n}$ of closed hyper-rectangular subsets of $\mathbb{R}^{n}$, i.e., regions of the form $\prod_{i=1}^{n} C_{i}$, where $C_{1}, \ldots, C_{n}$ are non-singleton closed intervals in $\mathbb{R}$, for some $n \geq 1$.

Sometimes, regions are required to satisfy additional constraints such as being connected or homeomorphic to the closed unit disc.

Given a topological space $\mathfrak{T}$ and a set of regions $U_{\mathfrak{T}}$, we define the extension of the eight Egenhofer-Franzosa (or RCC8) relations dc ('disconnected'), ec ('externally connected'), tpp ('tangential proper part'), tppi ('inverse of tangential proper part'), po ('partial overlap'), eq ('equal'), ntpp ('non-tangential proper part'), and nttpi ('inverse of non-tangential proper part') as the following subsets of $U_{\mathfrak{T}} \times U_{\mathfrak{T}}$ :

$$
\begin{array}{lll}
(s, t) \in \mathrm{dc}^{\mathfrak{T}} & \text { iff } & s \cap t=\emptyset \\
(s, t) \in \mathrm{ec}^{\mathfrak{T}} & \text { iff } & \mathbb{I}(s) \cap \mathbb{I}(t)=\emptyset \wedge s \cap t \neq \emptyset \\
(s, t) \in \mathrm{po}^{\mathfrak{T}} & \text { iff } & \mathbb{I}(s) \cap \mathbb{I}(t) \neq \emptyset \wedge s \nsubseteq t \wedge t \nsubseteq s \\
(s, t) \in \mathrm{eq}^{\mathfrak{T}} & \text { iff } & s=t
\end{array}
$$

\footnotetext{
${ }^{1}$ Another possibility is to identify regions with non-empty regular open sets instead of non-empty regular closed ones. The results presented in this paper hold for this alternative definition of regions as well.
} 


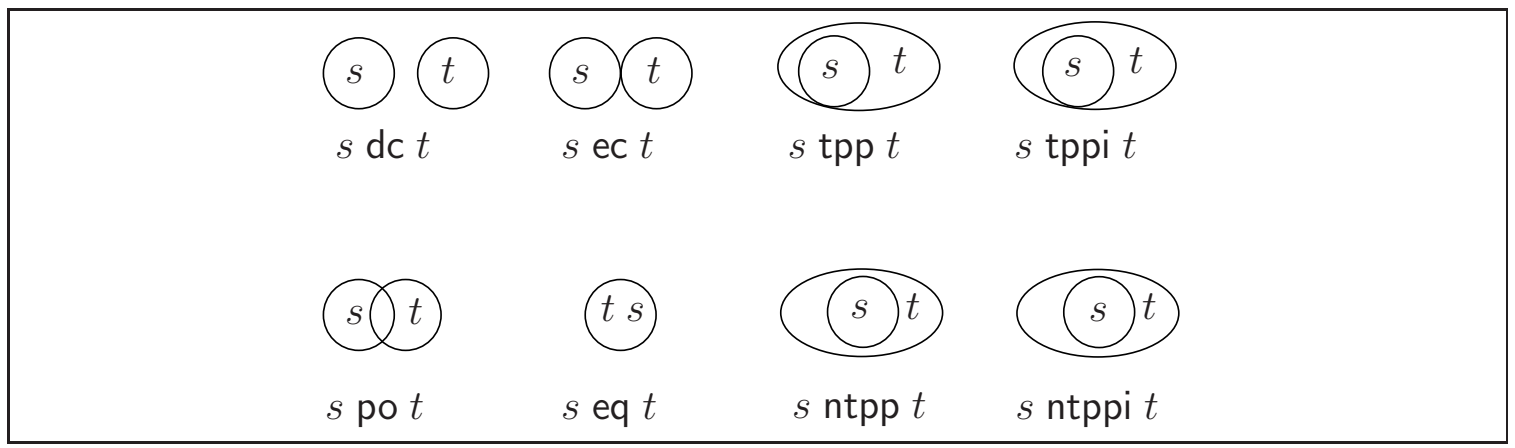

Figure 1: The eight relations between regions.

$$
\begin{array}{rll}
(s, t) \in \mathrm{tpp}^{\mathfrak{T}} & \text { iff } & s \subseteq t \wedge s \nsubseteq \mathbb{I}(t) \wedge s \neq t \\
(s, t) \in \mathrm{ntpp}^{\mathfrak{T}} & \text { iff } & s \subseteq \mathbb{I}(t) \wedge s \neq t \\
(s, t) \in \mathrm{tppi}^{\mathfrak{T}} & \text { iff } & (t, s) \in \mathrm{tpp}^{\mathfrak{T}} \\
(s, t) \in \mathrm{ntppi}^{\mathfrak{T}} & \text { iff } & (t, s) \in \mathrm{ntpp}^{\mathfrak{T}} .
\end{array}
$$

Figure 1 shows examples of the RCC8 relations in the real plane $\mathbb{R}^{2}$. The structure $\mathfrak{R}\left(\mathfrak{T}, U_{\mathfrak{T}}\right):=\left\langle U_{\mathfrak{T}}, \mathrm{dc}^{\mathfrak{T}}, \mathrm{ec}^{\mathfrak{T}}, \mathrm{po}^{\mathfrak{T}}, \mathrm{eq}^{\mathfrak{T}}, \mathrm{tpp}^{\mathfrak{T}}, \mathrm{ntpp}^{\mathfrak{T}}, \mathrm{tppi}^{\mathfrak{T}}, \mathrm{ntppi}^{\mathfrak{T}}\right\rangle$ is called the concrete region structure induced by $\left(\mathfrak{T}, U_{\mathfrak{T}}\right)$. Observe that concrete region structures do not include a valuation of propositional letters, and thus correspond to a frame in standard modal logic. We will later extend region structures to region models by augmenting them with valuation functions.

We now develop a first-order characterization of concrete region structures. This will establish some fundamental properties of concrete region structures that are used throughout the whole paper, and will also provide us with an easy proof of the fact that certain logics considered in this paper are recursively enumerable. We call a relational structure

$$
\mathfrak{R}=\left\langle W, \mathrm{dc}^{\mathfrak{R}}, \mathrm{ec}^{\mathfrak{R}}, \mathrm{po}^{\mathfrak{R}}, \mathrm{eq}^{\mathfrak{R}}, \mathrm{tpp}^{\mathfrak{R}}, \text { ntpp }^{\mathfrak{R}}, \text { tppi }^{\mathfrak{R}}, \text { ntppi }^{\mathfrak{R}}\right\rangle
$$

a general region structure if $W$ is a non-empty set and the $r^{\mathfrak{R}}$ are binary relations on $W$ that are mutually disjoint (i.e., $r^{\Re} \cap q^{\Re}=\emptyset$, for $r \neq q$ ), jointly exhaustive (i.e., the union of all $r^{\Re}$ is $W \times W$ ), and satisfy the following:

- eq is interpreted as the identity on $W, \mathrm{dc}^{\Re}, \mathrm{ec}^{\mathfrak{R}}$, and $\mathrm{po}^{\mathfrak{R}}$ are symmetric, and tppi $\mathrm{i}^{\mathfrak{R}}$ and ntppi ${ }^{\Re}$ are the inverse relations of $\operatorname{ttp}^{\Re}$ and $n t p p^{\Re}$, respectively;

- the rules of the composition table (Figure 2) are satisfied in the sense that, for any entry $\mathrm{q}_{1}, \ldots, \mathrm{q}_{k}$ in row $\mathrm{r}_{1}$ and column $\mathrm{r}_{2}$, the first-order sentence

$$
\forall x \forall y \forall z\left(\left(\mathrm{r}_{1}(x, y) \wedge \mathrm{r}_{2}(y, z)\right) \rightarrow\left(\mathrm{q}_{1}(x, z) \vee \cdots \vee \mathrm{q}_{k}(x, z)\right)\right.
$$

is valid ( $*$ is the disjunction over all eight relations).

The following theorem shows that, in some sense, concrete region structures and general region structures are interchangable. In what follows, we will thus often only speak of region structures and only distinguish between general and concrete region structures when necessary. A proof can be found in Appendix $\mathrm{A}$ 


\begin{tabular}{|c|c|c|c|c|c|c|c|}
\hline o & dc & ec & tpp & tppi & po & ntpp & ntppi \\
\hline dc & * & \begin{tabular}{|c|}
$\mathrm{dc}, \mathrm{ec}$ \\
$\mathrm{po}, \mathrm{tpp}$ \\
$\mathrm{ntpp}$
\end{tabular} & $\begin{array}{c}\text { dc,ec, } \\
\text { po,tpp, } \\
\text { ntpp }\end{array}$ & dc & $\begin{array}{c}\text { dc,ec, } \\
\text { po,tpp, } \\
\text { ntpp }\end{array}$ & $\begin{array}{c}\mathrm{dc}, \mathrm{ec} \\
\text { po,tpp, } \\
\text { ntpp }\end{array}$ & dc \\
\hline ec & $\begin{array}{c}\text { dc,ec, } \\
\text { po,tppi, } \\
\text { ntppi }\end{array}$ & \begin{tabular}{|c|}
$\mathrm{dc}, \mathrm{ec}$, \\
$\mathrm{po}, \mathrm{tpp}$, \\
tppi,eq
\end{tabular} & $\begin{array}{l}\text { ec,po, } \\
\text { tpp, } \\
\text { ntpp }\end{array}$ & $\mathrm{dc}, \mathrm{ec}$ & $\begin{array}{c}\text { dc,ec, } \\
\text { po,tpp, } \\
\text { ntpp }\end{array}$ & $\begin{array}{c}\text { po, } \\
\text { tpp, } \\
\text { ntpp }\end{array}$ & dc \\
\hline tpp & dc & $\mathrm{dc}, \mathrm{ec}$ & tpp,ntpp & $\begin{array}{l}\mathrm{dc}, \mathrm{ec}, \\
\text { po,tpp, } \\
\text { tppi,eq }\end{array}$ & $\begin{array}{c}\text { dc,ec, } \\
\text { po,tpp, } \\
\text { ntpp }\end{array}$ & ntpp & $\begin{array}{c}\mathrm{dc}, \mathrm{ec}, \\
\text { po,tppi, } \\
\text { ntppi }\end{array}$ \\
\hline tppi & $\begin{array}{c}\text { dc,ec, } \\
\text { po,tppi, } \\
\text { ntppi }\end{array}$ & $\begin{array}{l}\text { ec,po, } \\
\text { tppi, } \\
\text { ntppi }\end{array}$ & $\begin{array}{l}\text { po,eq, } \\
\text { tpp, } \\
\text { tppi }\end{array}$ & tppi,ntppi & $\begin{array}{l}\text { po, } \\
\text { tppi, } \\
\text { ntppi }\end{array}$ & $\begin{array}{l}\text { po, } \\
\text { tpp, } \\
\text { ntpp }\end{array}$ & ntppi \\
\hline po & $\begin{array}{c}\text { dc,ec, } \\
\text { po,tppi, } \\
\text { ntppi }\end{array}$ & \begin{tabular}{|c|} 
dc,ec, \\
po,tppi, \\
ntppi
\end{tabular} & $\begin{array}{c}\text { po, } \\
\text { tpp, } \\
\text { ntpp }\end{array}$ & $\begin{array}{c}\text { dc,ec, } \\
\text { po,tppi, } \\
\text { ntppi }\end{array}$ & $*$ & $\begin{array}{c}\text { po, } \\
\text { tpp, } \\
\text { ntpp }\end{array}$ & $\begin{array}{c}\text { dc,ec, } \\
\text { po,tppi, } \\
\text { ntppi }\end{array}$ \\
\hline ntpp & dc & dc & ntpp & $\begin{array}{l}\text { dc,ec, } \\
\text { po,tpp, } \\
\text { ntpp }\end{array}$ & $\begin{array}{c}\text { dc,ec, } \\
\text { po,tpp, } \\
\text { ntpp }\end{array}$ & ntpp & $*$ \\
\hline ntppi & $\begin{array}{c}\mathrm{dc}, \mathrm{ec}, \\
\text { po,tppi, } \\
\text { ntppi }\end{array}$ & $\begin{array}{l}\text { po, } \\
\text { tppi, } \\
\text { ntppi }\end{array}$ & $\begin{array}{l}\text { po, } \\
\text { tppi, } \\
\text { ntppi }\end{array}$ & ntppi & $\begin{array}{l}\text { po, } \\
\text { tppi, } \\
\text { ntppi }\end{array}$ & $\begin{array}{l}\text { po, tppi, } \\
\text { tpp, ntpp, } \\
\text { ntppi,eq }\end{array}$ & ntppi \\
\hline
\end{tabular}

Figure 2: The composition table.

Theorem 1 (Representation theorem).

(i) Every concrete region structure is a general region structure;

(ii) every general region structure is isomorphic to a concrete region structure;

(iii) for every $n>0$, every countable general region structure is isomorphic to a concrete region structure of the form $\mathfrak{R}\left(\mathbb{R}^{n}, U_{\mathbb{R}^{n}}\right)$ (with $U_{\mathbb{R}^{n}} \subseteq \mathbb{R}_{\text {reg }}^{n}$ ).

Note that Points (ii) and (iii) of Theorem 1 rely on the fact that we admit any non-empty set of non-empty regular closed sets as a possible choice for the regions of a topological space. This is of course different from admitting only structures in which, for example, all non-empty regular closed sets are required to be regions, or all closed hyper-rectangles are required to be regions. The logics introduced in Section 4 will be based on both kinds of structures. Quite informally, we shall in the following call structures of the latter kind full concrete region structures. We introduce some useful classes of region structures:

- $\mathcal{R S}$ is the class of all general region structures;

- $\mathcal{T O P}$ denotes the class of all region structures $\mathfrak{R}\left(\mathfrak{T}, \mathfrak{T}_{\text {reg }}\right)$.

Observe that the structures in $\mathcal{T O P}$ are full concrete region structures. It is interesting to note that, in contrast to $\mathcal{R S}, \mathcal{T} \mathcal{O P}$ cannot be characterized by means of a recursively enumerable set of first-order sentences. This follows from the non-recursive enumerability of the logic of $\mathcal{T O P}$ to be introduced and investigated later.

We should also note that the region structure $\mathfrak{R}\left(\mathbb{R}, \mathbb{R}_{\text {rect }}\right)=\mathfrak{R}\left(\mathbb{R}, \mathbb{R}_{\text {conv }}\right)$ is an interval structure. Therefore, topological modal logics interpreted in such structures may be viewed as temporal interval logics similar to the ones defined by Halpern and Shoham in HS91. A minor technical difference between our interval structure and the ones considered by Halpern and Shoham is that our requirement of regular closedness excludes point-intervals, while such intervals are admitted by Halpern and Shoham. 


\section{The Language}

The modal language $\mathcal{L}_{\mathrm{RCC} 8}$ extends propositional logic with countably many variables $p_{1}, p_{2}, \ldots$ and the Boolean connectives $\neg$ and $\wedge$ by means of the unary modal operators [dc], [ec], etc. (one for each topological relation). A region model $\mathfrak{M}=\left\langle\mathfrak{R}, p_{1}^{\mathfrak{M}}, p_{2}^{\mathfrak{M}}, \ldots\right\rangle$ for $\mathcal{L}_{\mathrm{RCC} 8}$ consists of a region structure $\mathfrak{R}=\left\langle W, \mathrm{dc} c^{\mathfrak{R}}, \mathrm{ec}^{\mathfrak{R}}, \ldots\right\rangle$ and the interpretation $p_{i}^{\mathfrak{M}}$ of the variables $p_{i}$ of $\mathcal{L}_{\mathrm{RCC} 8}$ as subsets of $W$. A formula $\varphi$ is either true at a region $s \in W$ (written $\mathfrak{M}, s \models \varphi$ ) or false at $s$ (written $\mathfrak{M}, s \not \models \varphi$ ), the inductive definition being as follows:

(1) if $\varphi$ is a prop. variable, then $\mathfrak{M}, s \models \varphi$ iff $s \in \varphi^{\mathfrak{M}}$;

(2) $\mathfrak{M}, s \models \neg \varphi$ iff $\mathfrak{M}, s \not \models \varphi$;

(3) $\mathfrak{M}, s \models \varphi_{1} \wedge \varphi_{2}$ iff $\mathfrak{M}, s \models \varphi_{1}$ and $\mathfrak{M}, s \models \varphi_{2}$;

(4) $\mathfrak{M}, s \models[r] \varphi$ iff, for all $t \in W,(s, t) \in \mathrm{r}^{\mathfrak{R}}$ implies $\mathfrak{M}, t \models \varphi$.

We use the usual abbreviations: $\varphi \rightarrow \psi$ for $\neg \varphi \vee \psi$ and $\langle\mathrm{r}\rangle \varphi$ for $\neg[\mathrm{r}] \neg \varphi$.

In the remainder of this section, we discuss the expressive power of the language $\mathcal{L}_{\mathrm{RCC}}$. The discussion starts with some simple observations.

- First, the difference modality $\square_{d} \varphi$, investigated for example in dR92, has the following semantics:

$$
\mathfrak{M}, s \models \square_{d} \varphi \text { iff } \mathfrak{M}, t \models \varphi \text { for all } t \in W \text { such that } t \neq s .
$$

In $\mathcal{L}_{\mathrm{RCC} 8}$, it can be expressed as $\bigwedge_{\mathrm{r} \in \mathrm{RCC}-\{\mathrm{eq}\}}[\mathrm{r}] \varphi$ since the relations are jointly exhaustive and mutually exclusive.

- Second, the useful universal box $\square_{u} \varphi$, which is well-known from modal logic GP92, has the following semantics:

$$
\mathfrak{M}, s \models \square_{u} \varphi \text { iff } \mathfrak{M}, t \models \varphi \text { for all } t \in W .
$$

In $\mathcal{L}_{\mathrm{RCC} 8}$, it can be expressed as $\varphi \wedge \square_{d} \varphi$.

- Third, we can express that a formula $\varphi$ holds in precisely one region (i.e., is $a$ nominal GV93) by writing

$$
\operatorname{nom}(\varphi)=\diamond_{u}\left(\varphi \wedge \square_{d} \neg \varphi\right)
$$

where $\diamond_{u} \varphi=\neg \square_{u} \neg \varphi$. The availability of nominals means that we can introduce names for regions; e.g., the formulas

$$
\operatorname{nom}(\text { Elbe }), \quad \operatorname{nom}(\text { Dresden })
$$

state that "Elbe" (the name of a river) and "Dresden" each apply to exactly one region.

- Finally, it is often useful to define operators [pp] and [ppi] as abbreviations:

$$
\begin{aligned}
{[\mathrm{pp}] \varphi } & =[\mathrm{tpp}] \varphi \wedge[\mathrm{nttp}] \varphi \\
{[\mathrm{ppi}] \varphi } & =[\mathrm{tppi}] \varphi \wedge[\text { nttpi }] \varphi .
\end{aligned}
$$

As in the temporal case HS91 and following Cohn Coh93, we can use these new operators to classify formulas $\varphi$ according to whether

- they are homogeneous, i.e. they hold continuously throughout regions:

$$
\square_{u}(\varphi \rightarrow[\mathrm{pp}] \varphi)
$$

- they are anti-homogeneous, i.e. they hold only in regions whose interiors are mutually disjoint:

$$
\square_{u}(\varphi \rightarrow([\mathrm{pp}] \neg \varphi \wedge[\mathrm{po}] \neg \varphi)
$$


Instances of anti-homogeneous propositions are "river" and "university campus", while "occupied-by-water" is homogeneous.

As this paper concentrates on the investigation of the expressivity and computational properties of topological modal logics, it is out of scope to describe potential applications in detail. Therefore, we only give a few illustrative examples of statements in $\mathcal{L}_{\text {RCC8 }}$. The following example describes, in a drastically simplified way, the relationship of cities, harbours, rivers, and the sea. Based on this 'background theory', it then describes the relationship of the city of Dresden and the river Elbe.

$$
\begin{aligned}
& \square_{u}(\text { harbor-city } \leftrightarrow(\text { city } \wedge\langle\mathrm{ppi}\rangle \text { harbor })) \\
& \square_{u}(\text { harbor } \rightarrow(\langle\mathrm{ec}\rangle \text { river } \vee\langle\mathrm{ec}\rangle \text { sea })) \\
& \square_{u}(\text { Dresden } \rightarrow \text { harbor-city }) \\
& \square_{u}(\text { Elbe } \rightarrow \text { river }) \\
& \square_{u}\left(\text { Dresden } \rightarrow \bigwedge_{\mathrm{r} \in \mathrm{RCC} 8-\{\mathrm{dc}\}}[\mathrm{r}] \neg \text { sea }\right) \\
& \square_{u}\left(\text { Dresden } \rightarrow\left(\langle\mathrm{po}\rangle \text { Elbe } \wedge \bigwedge_{\mathrm{r} \in \mathrm{RCC} 8-\{\mathrm{dc}\}}[\mathrm{r}](\text { river } \rightarrow \text { Elbe })\right)\right)
\end{aligned}
$$

From these formulas, it follows that Dresden has a part that is a harbor and is related via ec to the river Elbe.

The example suggests a scheme for the representation of spatial knowledge in $\mathcal{L}_{\mathrm{RCC}}$ that is known from description logic $\mathrm{BCM}^{+} 03$ : a background theory (called TBox in description logic) represents knowledge about general classes of regions such as those describing harbors and rivers. Knowledge about particular regions is formulated by using nominals and expressing spatial relations between them. In description logic, knowledge of this latter kind would be stored in an ABox.

We now relate the expressive power of the modal language $\mathcal{L}_{\mathrm{RCC} 8}$ to the expressive power of two standard formalisms for spatial reasoning: constraint networks and spatial first-order theories.

RCC8 constraint networks are a basic, but rather popular formalism for representing spatial knowledge using the RCC8 relations RN99, Ege94, GPP95, SS01, RCC92]. In the following, we show that our modal language $\mathcal{L}_{\mathrm{RCC} 8}$ can capture constraint networks in a straightforward way. An RCC8 constraint network is a finite set of constraints $(s \mathrm{r} r)$ with $s, r$ region variables and $r$ an RCC8 relation. Such a network $N$ is satisfiable in a topological space $\mathfrak{T}$ with regions $U_{\mathfrak{T}}$ if there exists an assignment $\delta$ of regions in $U_{\mathfrak{T}}$ to region variables such that $(s \mathrm{r} r) \in N$ implies $\delta(s) \mathrm{r}^{\mathfrak{T}} \delta(r)$. In our language $\mathcal{L}_{\mathrm{RCC} 8}$, we can express a constraint network $N$ that uses region variables $s_{1}, \ldots, s_{k}$ by writing

$$
\bigwedge_{\left(s_{i} r s_{j}\right) \in N} \diamond_{u}\left(p_{i} \wedge\langle\mathrm{r}\rangle p_{j}\right) \wedge \bigwedge_{1 \leq i \leq k} \operatorname{nom}\left(p_{i}\right)
$$

This formula is clearly satisfiable iff $N$ is satisfiable.

Spatial first-order theories are usually formulated in first-order languages equivalent to the first-order language $\mathcal{F} \mathcal{O}_{\mathrm{RCC} 8}$ that has equality, eight binary predicates for the RCC8 relations, no function symbols, and no unary predicates PSV99, PS98, SS01, RCC92. Intuitively, we cannot reduce $\mathcal{L}_{\mathrm{RCC}}$ to such languages because they do not offer a counterpart of $\mathcal{L}_{\mathrm{RCC}}$ 's propositional letters. A formal proof is provided by the following two observations:

(1) $\mathcal{F} \mathcal{O}_{\mathrm{RCC}}$ is decidable over the region structure $\mathfrak{R}\left(\mathbb{R}^{2}, \mathbb{R}_{\text {rect }}^{2}\right)$. Indeed, it is not hard to verify that there is a reduction to the first-order theory of $\langle\mathbb{R},<\rangle$ which coincides with the first-order theory of $\langle\mathbb{Q},<\rangle$ and, therefore, is decidable End72. Details 
of the reduction are omitted as it is similar to the proof of Theorem 14 given in Appendix C (but simpler).

(2) In Section 6, we show that $\mathcal{L}_{\mathrm{RCC}}$ is not recursively enumerable over $\mathfrak{R}\left(\mathbb{R}^{2}, \mathbb{R}_{\text {rect }}^{2}\right)$. Thus, the adequate first-order language to compare $\mathcal{L}_{\mathrm{RCC} 8}$ with is the monadic extension $\mathcal{F} \mathcal{O}_{\mathrm{RCC} 8}^{m}$ of $\mathcal{F} O_{\mathrm{RCC} 8}$ that is obtained by adding countably many unary predicates $p_{1}, p_{2}, \ldots$ By well-known results from modal correspondence theory Gab81b, any $\mathcal{L}_{\mathrm{RCC} 8}$ formula $\varphi$ can be polynomially translated into a formula $\varphi^{*}$ of $\mathcal{F} \mathcal{O}_{\mathrm{RCC}}^{m}$ with only two variables such that, for any region model $\mathfrak{M}$ and any region $s$,

$$
\mathfrak{M}, s \models \varphi \text { iff } \mathfrak{M} \models \varphi^{*}[s] .
$$

More surprisingly, the converse holds as well: this follows from recent results of [LSW01] since the RCC8 relations are mutually exclusive and jointly exhaustive. A proof sketch of the following theorem can be found in Appendix B

Theorem 2. For every $\mathcal{F O}_{\mathrm{RCC}}^{m}$-formula $\varphi(x)$ with free variable $x$ that uses only two variables, one can effectively construct a $\mathcal{L}_{\mathrm{RCC} 8}$-formula $\varphi^{*}$ of length at most exponential in the length of $\varphi(x)$ such that, for every region model $\mathfrak{M}$ and any region $s, \mathfrak{M}, s \models \varphi^{*}$ iff $\mathfrak{M} \models$ $\varphi[s]$.

However, there is also an important difference between $\mathcal{L}_{\mathrm{RCC}}$ and the two-variable fragment of $\mathcal{F O}_{\mathrm{RCC} 8}^{m}$ : the latter is exponentially more succinct than the former. This can be shown using a formula proposed by Etessami, Vardi, and Wilke EVW02 stating that any two regions agreeing on $p_{0}, \ldots, p_{n-1}$ also agree on $p_{n}$. A proof can be found in Appendix B.

Theorem 3. For $n \geq 1$, define a $\mathcal{F O}_{\mathrm{RCC} 8}^{m}$ formula

$$
\varphi_{n}:=\forall x \forall y\left(\bigwedge_{i<n}\left(p_{i}(x) \leftrightarrow p_{i}(y)\right) \rightarrow\left(p_{n}(x) \leftrightarrow p_{n}(y)\right)\right)
$$

Then every $\mathcal{L}_{\mathrm{RCC} 8}$-formula $\psi_{n}$ that is equivalent to $\varphi_{n}$ on the class of all region structures $\mathcal{R S}$ has length $2^{\Omega(n)} .^{2}$

We believe that this succinctness result also holds on other classes of region structures such as the singleton $\left\{\mathfrak{R}\left(\mathbb{R}^{n}, \mathbb{R}_{\text {reg }}^{n}\right)\right\}$, but leave the proof as an open problem.

\section{LOGICS}

In this section, we define a number of topological modal logics by applying the language $\mathcal{L}_{\mathrm{RCC}}$ to different classes of region structures. We also establish a number of separation results showing that logics obtained from different classes of region structures do not usually coincide.

Let $\mathcal{S}$ be a class of region structures. An $\mathcal{L}_{\mathrm{RCC}}$ formula $\varphi$ is valid in $\mathcal{S}$ if it is true in all regions of all models based on region structures from $\mathcal{S}$. We use $L_{\mathrm{RCC}}(\mathcal{S})$ to denote the logic of $\mathcal{S}$, i.e., the set of all $\mathcal{L}_{\mathrm{RCC} 8}$-formulas valid in $\mathcal{S}$. If $\mathcal{S}=\left\{\mathfrak{R}\left(\mathfrak{T}, U_{\mathfrak{T}}\right)\right\}$ for some topological space $\mathfrak{T}$ with regions $U_{\mathfrak{T}}$, then we abbreviate $L_{\mathrm{RCC} 8}(\mathcal{S})$ by writing $L_{\mathrm{RCC} 8}\left(\mathfrak{T}, U_{\mathfrak{T}}\right)$. The following logics of full concrete region structures (see Section 2) will play a prominent role in this paper:

\footnotetext{
${ }^{2}$ Following the formulation of Theorem 2 the formula $\psi_{n}$ is called equivalent to $\varphi_{n}$ if the following holds: for every region model $\mathfrak{M}$ and any region $s, \mathfrak{M}, s \models \psi_{n}$ iff $\mathfrak{M} \models \varphi_{n}[s]$. As the formula $\varphi_{n}$ does not have a free variable, the right hand side of this equivalence does not depend on $s$.
} 
- the logic $L_{\mathrm{RCC} 8}(\mathcal{T O P})$ of all full concrete region structures of regular closed regions $\mathfrak{R}\left(\mathfrak{T}, \mathfrak{T}_{\text {reg }}\right)$;

- logics based on the $\mathbb{R}^{n}$, for some $n \geq 1$ : $L_{\mathrm{RCC} 8}\left(\mathbb{R}^{n}, \mathbb{R}_{\text {reg }}^{n}\right), L_{\mathrm{RCC} 8}\left(\mathbb{R}^{n}, \mathbb{R}_{\text {conv }}^{n}\right)$, and $L_{\mathrm{RCC} 8}\left(\mathbb{R}^{n}, \mathbb{R}_{\text {rect }}^{n}\right)$.

We will also study the logic $L_{\mathrm{RCC}}(\mathcal{R S})$ of all region structures. Note that the region classes underlying the above logics admit unbounded regions such as $\mathbb{R}^{n}$. However, the technical results proved in this paper also hold if we consider bounded regions, only.

We now investigate the relationship between the introduced logics. As an exhaustive analysis is out of the scope of this paper, we only treat some important cases:

(1) $L_{\mathrm{RCC} 8}(\mathcal{T} \mathcal{O P}) \nsubseteq L_{\mathrm{RCC} 8}(\mathcal{R} S)$ and $L_{\mathrm{RCC} 8}\left(\mathbb{R}^{n}, \mathbb{R}_{\times}^{n}\right) \nsubseteq L_{\mathrm{RCC} 8}(\mathcal{R S})$ for $\mathrm{x} \in\{$ reg, conv, rect $\}$ and $n>0$ since

$$
\left(\operatorname{nom}(p) \wedge \operatorname{nom}(q) \wedge \diamond_{u}(p \wedge\langle\mathrm{dc}\rangle q)\right) \rightarrow \diamond_{u}(\langle\mathrm{ppi}\rangle p \wedge\langle\mathrm{ppi}\rangle q)
$$

is not valid in $\mathcal{R S}$ (it states that any two disconnected regions are proper parts of a region). The converse inclusions obviously hold for all $n>0$.

(2) $L_{\mathrm{RCC} 8}\left(\mathbb{R}^{n}, \mathbb{R}_{\times}^{n}\right) \nsubseteq L_{\mathrm{RCC} 8}(\mathcal{T O P})$ for $\mathrm{x} \in\{$ reg, conv, rect $\}$ and $n>0:\langle\mathrm{ppi}\rangle \top$ is valid in $\mathfrak{R}\left(\mathbb{R}^{n}, \mathbb{R}_{x}^{n}\right)$, but not in $\mathcal{T O P}$. For the converse direction, we clearly have $L_{\mathrm{RCC} 8}(\mathcal{T O P}) \subseteq L_{\mathrm{RCC} 8}\left(\mathbb{R}^{n}, \mathbb{R}_{\text {reg }}^{n}\right)$ for all $n>0$.

(3) For $n, m>0$ and $m^{\prime}>1, L_{\mathrm{RCC} 8}\left(\mathbb{R}^{n}, \mathbb{R}_{\text {rect }}^{n}\right) \nsubseteq L$, where $L$ is any logic from $L_{\mathrm{RCC} 8}\left(\mathbb{R}^{n+1}, \mathbb{R}_{\text {rect }}^{n+1}\right), L_{\mathrm{RCC} 8}\left(\mathbb{R}^{m^{\prime}}, \mathbb{R}_{\text {conv }}^{m^{\prime}}\right), L_{\mathrm{RCC} 8}\left(\mathbb{R}^{m}, \mathbb{R}_{\text {reg }}^{m}\right), L_{\mathrm{RCC} 8}(\mathcal{T} \mathcal{O P}), L_{\mathrm{RCC} 8}(\mathcal{R S})$. To see this define, for $k>0$, an RCC8 constraint network ec $[k]$ as follows:

$$
\text { ec }[k]=\left\{\left(x_{i} \text { ec } x_{j}\right) \mid 1 \leq i, j \leq k\right\} .
$$

For $n>0$, ec $\left[2^{n}+1\right]$ is not satisfiable in $\mathfrak{R}\left(\mathbb{R}^{n}, \mathbb{R}_{\text {rect }}^{n}\right)$, but it is satisfiable in the classes of region structures determining the logics $L$. Observe that the condition $m^{\prime}>1$ is required because $\mathfrak{R}\left(\mathbb{R}, \mathbb{R}_{\text {conv }}\right)=\mathfrak{R}\left(\mathbb{R}, \mathbb{R}_{\text {rect }}\right)$.

(4) For $n>0, L_{\mathrm{RCC} 8}\left(\mathbb{R}^{n}, \mathbb{R}_{\text {conv }}^{n}\right) \nsubseteq L_{\mathrm{RCC} 8}\left(\mathbb{R}^{n+1}, \mathbb{R}_{\text {conv }}^{n+1}\right)$. Since $L_{\mathrm{RCC} 8}\left(\mathbb{R}, \mathbb{R}_{\text {conv }}\right)=$ $L_{\mathrm{RCC} 8}\left(\mathbb{R}, \mathbb{R}_{\text {rect }}\right)$, the case $n=1$ follows from the previous item. Regarding the cases $n>1$, for simplicity we only consider $n=2$ explicitly. A generalization is straightforward. Take region variables $x_{i j}, 1 \leq i<j \leq 4$. Then the constraint network obtained as the union of ec[4],

$$
\left\{\left(x_{i} \text { pp } x_{i j}\right),\left(x_{j} \text { pp } x_{i j}\right) \mid 1 \leq i<j \leq 4\right\}
$$

and

$$
\left\{\left(x_{i j} \text { ec } x_{k}\right) \mid 1 \leq i<j \leq 4, k \in\{1,2,3,4\}-\{i, j\}\right\}
$$

is satisfiable in $\Re\left(\mathbb{R}^{3}, \mathbb{R}_{\text {conv }}^{3}\right)$ but not in $\mathfrak{R}\left(\mathbb{R}^{2}, \mathbb{R}_{\text {conv }}^{2}\right)$.

(5) For all $n, m>0, L_{\mathrm{RCC} 8}\left(\mathbb{R}^{n}, \mathbb{R}_{\text {reg }}^{n}\right) \nsubseteq L_{\mathrm{RCC} 8}\left(\mathbb{R}^{m}, \mathbb{R}_{\text {conv }}^{m}\right)$ and $L_{\mathrm{RCC} 8}\left(\mathbb{R}^{n}, \mathbb{R}_{\text {reg }}^{n}\right) \nsubseteq$ $L_{\mathrm{RCC} 8}\left(\mathbb{R}^{m}, \mathbb{R}_{\text {rect }}^{m}\right)$ : the following formula states that, for any three pair-wise disconnected regions, there is another region containing only the first two (but not the third) as a proper part:

$$
\begin{aligned}
\left(\bigwedge_{1 \leq i \leq 3} \operatorname{nom}\left(p_{i}\right) \wedge \bigwedge_{\substack{1 \leq i<j \leq 3 \\
\diamond_{u}\left(\langle\mathrm{ppi}\rangle p_{1} \wedge\langle\mathrm{ppi}\rangle p_{2} \wedge \neg\langle\mathrm{ppi}\rangle p_{3}\right) .}} \diamond_{u}\left(p_{i} \wedge\langle\mathrm{dc}\rangle p_{j}\right)\right) \rightarrow
\end{aligned}
$$

This formula is valid in $\mathfrak{R}\left(\mathbb{R}^{n}, \mathbb{R}_{\text {reg }}^{n}\right)$, but not in $\mathfrak{R}\left(\mathbb{R}^{n}, \mathbb{R}_{\text {conv }}^{n}\right)$ and $\mathfrak{R}\left(\mathbb{R}^{n}, \mathbb{R}_{\text {rect }}^{n}\right)$. 
As these examples show, $\mathcal{L}_{\mathrm{RCC}}$ is powerful enough to "feel" the difference between different topological spaces and different choices of regions.

While full concrete region structures are appropriate for reasoning about topological spaces themselves, for many applications it is not adequate to demand that models have to comprise all regions of a particular form (such as the non-empty regular closed ones or the closed hyper-rectangles). In such applications, models may contain only some such regions - those that are inhabited by spatial objects that are relevant for the application. This observation gives rise to another class of topological modal logics: given a class $\mathcal{S}$ of region structures, we use $L_{\mathrm{RCC} 8}^{\mathrm{S}}(\mathcal{S})$ to denote the logic determined by the class of all substructures of structures in $\mathcal{S}$. Note that the class $\mathcal{R S}$ is closed under substructures by definition, and thus we have $L_{\mathrm{RCC} 8}(\mathcal{R S})=L_{\mathrm{RCC} 8}^{\mathrm{S}}(\mathcal{R S})$. Taking this idea one step further, we may even be concerned with applications where the number of relevant spatial objects is known to be finite, but their exact number is unknown. Then, we should consider only models comprising a finite number of regions, without assuming an upper bound on their number. Thus, we use $L_{\mathrm{RCC} 8}^{\text {fin }}(\mathcal{S})$ to denote the logic of all finite substructures of structures in $\mathcal{S}$.

The inclusion of such substructure logics and their finite versions is a distinguishing feature of the undecidability results proved in this paper: the general undecidability theorems presented in Sections [5 and 7 cover all logics of full concrete region structures introduced in this section, as well as their substructure variants and finite substructure variants. In contrast, the undecidability proofs of Halpern and Shoham for interval temporal logics are not applicable to the substructure variants of these logics [HS91]. Moreover, it will turn out that logics of full concrete region structures are usually $\Pi_{1}^{1}$-hard, while their substructure counterparts are usually recursively enumerable.

We now continue our investigation of the relationship between topological modal logics, taking into account substructure logics and their finite companions. Some of the new family members turn out to be already known:

Theorem 4. For $n>0$, we have

(1) $L_{\mathrm{RCC} 8}(\mathcal{R S})=L_{\mathrm{RCC} 8}^{\mathrm{S}}(\mathcal{T} \mathcal{O P})=L_{\mathrm{RCC} 8}^{\mathrm{S}}\left(\mathbb{R}^{n}, \mathbb{R}_{\text {reg }}^{n}\right)$;

(2) $L_{\mathrm{RCC} 8}^{\mathrm{fin}}(\mathcal{R S})=L_{\mathrm{RCC} 8}^{\text {fin }}(\mathcal{T O P})=L_{\mathrm{RCC} 8}^{\text {fin }}\left(\mathbb{R}^{n}, \mathbb{R}_{\mathrm{reg}}^{n}\right)$.

Proof All the mentioned logics are modal logics determined by classes of structures that are closed under substructures. As shown in Wol97, Corollary 3.8, such modal logics are determined by the at most countable members of those classes. Thus, Theorem 4 is an immediate consequence of Theorem 1 .

A few additional interesting observations are the following:

(6) The non-inclusions given under Items 3 and 4 above also hold for the corresponding substructure and finite substructure cases. The proofs are identical.

(7) The arguments given in Items 1, 2 and 5 do not carry over since the given formulas are not valid in the corresponding substructures and finite substructures. Indeed, by Theorem 4 in these cases the first claim of Item 1 does not hold and the remaining claims of Item 1 and 2 do not hold for $\mathrm{x}=$ reg. In Item 5 , the statement is wrong in the substructure case and finite substructure case: it is not hard to see that, e.g., $L_{\mathrm{RCC} 8}^{\mathrm{S}}\left(\mathbb{R}^{n}, \mathbb{R}_{\text {reg }}^{n}\right) \subseteq L_{\mathrm{RCC} 8}^{\mathrm{S}}\left(\mathbb{R}^{n}, \mathbb{R}_{\text {conv }}^{n}\right)$ and $L_{\mathrm{RCC} 8}^{\mathrm{S}}\left(\mathbb{R}^{n}, \mathbb{R}_{\text {reg }}^{n}\right) \subseteq L_{\mathrm{RCC} 8}^{\mathrm{S}}\left(\mathbb{R}^{n}, \mathbb{R}_{\text {rect }}^{n}\right)$ for all $n>0$, and analogous claims hold in the finite substructure case. 


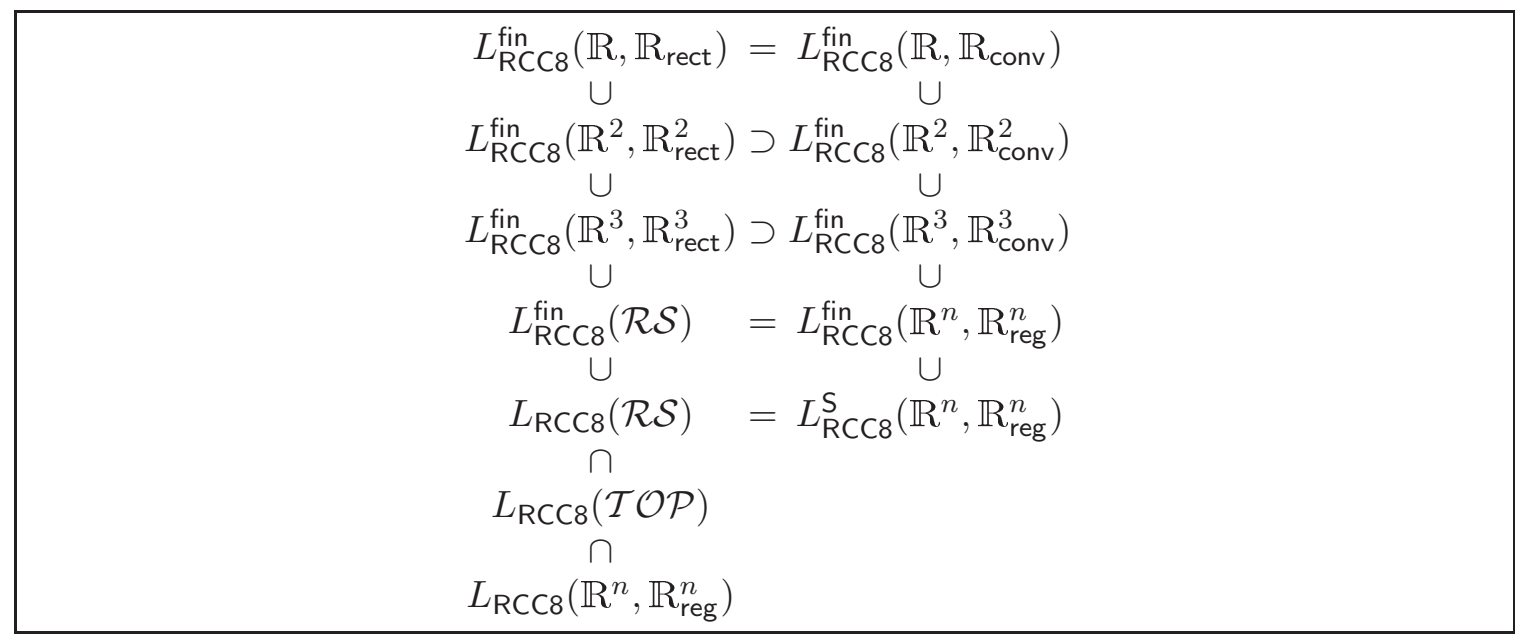

Figure 3: Inclusions between logics.

(8) $L_{\mathrm{RCC} 8}^{\text {fin }}(\mathcal{S}) \nsubseteq L$ for any class of region structures $\mathcal{S}$ and $L$ among $L_{\mathrm{RCC} 8}(\mathcal{R S})$, $L_{\mathrm{RCC} 8}(\mathcal{T} \mathcal{O P})$ and $L_{\mathrm{RCC} 8}\left(\mathbb{R}^{n}, U_{n}\right)$ with $n \geq 1$ and $\mathbb{R}_{\text {rect }}^{n} \subseteq U_{n}$ : the Löb-formula from modal logic

$$
[\mathrm{pp}]([\mathrm{pp}] p \rightarrow p) \rightarrow[\mathrm{pp}] p .
$$

is valid in a relational structure iff there is no infinite ascending pp-chain, see GKWZ03, pages 8-12. Thus, this formula is valid in all finite region structures, but not in all infinite ones.

(9) A number of additional inclusions is easily derived such as $L_{\mathrm{RCC} 8}^{\mathrm{S}}\left(\mathbb{R}^{n+1}, \mathbb{R}_{\text {rect }}^{n+1}\right) \subseteq$ $L_{\mathrm{RCC} 8}^{\mathrm{S}}\left(\mathbb{R}^{n}, \mathbb{R}_{\text {rect }}^{n}\right)$, for $n>0$ : it is easy to convert a substructure of $\mathfrak{R}\left(\mathbb{R}^{n+1}, \mathbb{R}_{\text {rect }}^{n+1}\right)$ into an isomorphic substructure of $\mathfrak{R}\left(\mathbb{R}^{n}, \mathbb{R}_{\text {rect }}^{n}\right)$.

The derived inclusions are summarized in Figure 3. By Points 1 to 9 above, all listed inclusions are indeed proper. For the sake of readability, we do not attempt to display all derived non-inclusions in Figure 3

\section{UNDECIDABILITY}

We now establish the central result of this paper: a rather general undecidability result that covers all logics introduced in the previous section. The only exceptions are logics based on classes of finite region structures, whose undecidability will be established in Section 7 To the best of our knowledge, the undecidability result proved in this section covers all classes of region structures that have been considered in the literature and contain at least one infinite structure. As the precise formulation of the result is somewhat technical, we start with a weaker version in which we require that the class of region structures contains at least one structure of the form $\mathfrak{R}\left(\mathbb{R}^{n}, U\right)$ with $\mathbb{R}_{\text {rect }}^{n} \subseteq U$. This condition will later be replaced with a more general one.

Theorem 5. Let $\mathcal{S} \subseteq \mathcal{R S}$ and suppose there exists $n>0$ and a set $U \subseteq \mathbb{R}_{\text {reg }}^{n}$ such that $\mathbb{R}_{\text {rect }}^{n} \subseteq U$ and $\mathfrak{R}\left(\mathbb{R}^{n}, U\right) \in \mathcal{S}$. Then $L_{\mathrm{RCC} 8}(\mathcal{S})$ is undecidable. 


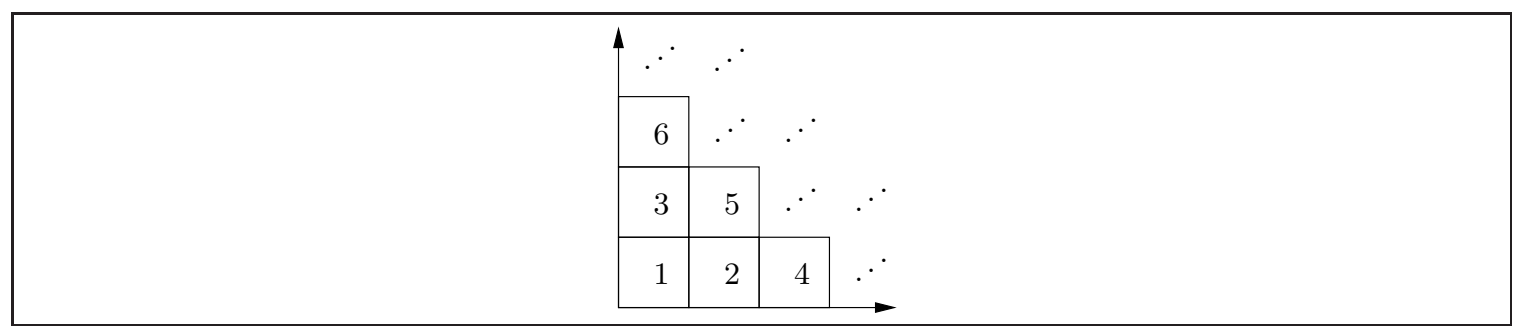

Figure 4: Enumerating tile positions.

Concerning the logics introduced in Section [4, we thus obtain the following:

Corollary 6. The logics $L_{\mathrm{RCC} 8}(\mathcal{S})$ and $L_{\mathrm{RCC} 8}^{\mathrm{S}}(\mathcal{S})$ are undecidable, for $\mathcal{S}$ one of $\mathcal{R S}, \mathcal{T} \mathcal{O P}$, $\mathfrak{R}\left(\mathbb{R}^{n}, \mathbb{R}_{\text {reg }}^{n}\right), \mathfrak{R}\left(\mathbb{R}^{n}, \mathbb{R}_{\text {conv }}^{n}\right)$, and $\mathfrak{R}\left(\mathbb{R}^{n}, \mathbb{R}_{\text {rect }}^{n}\right)$, with $n>0$.

We now develop the proof of Theorem [5. As we shall see, the proof suggests the mentioned generalization of Theorem [5] which will be stated subsequently. To ease notation, in the proofs given in this and the following sections we denote accessibility relations in models simply with dc, ec, etc., instead of with $\mathrm{dc}^{\mathfrak{R}}$, ec $\mathrm{c}^{\mathfrak{R}}$, etc.

The proof of Theorem $[5$ is by reduction of the domino problem that requires tiling of the first quadrant of the plane to the satisfiability of $\mathcal{L}_{\mathrm{RCC} 8}$ formulas. As usual, a formula $\varphi$ is called satisfiable in a region model $\mathfrak{M}=\left\langle W, \mathrm{dc}\right.$, ec $\left., \ldots, p_{1}^{\mathfrak{M}}, p_{2}^{\mathfrak{M}}, \ldots\right\rangle$ if there is an $s \in W$ with $\mathfrak{M}, s \models \varphi$.

Definition 7. Let $\mathcal{D}=(T, H, V)$ be a domino system, where $T$ is a finite set of tile types and $H, V \subseteq T \times T$ represent the horizontal and vertical matching conditions. We say that $\mathcal{D}$ tiles the first quadrant of the plane iff there exists a mapping $\tau: \mathbb{N}^{2} \rightarrow T$ such that, for all $(x, y) \in \mathbb{N}^{2}$ :

- if $\tau(x, y)=t$ and $\tau(x+1, y)=t^{\prime}$, then $\left(t, t^{\prime}\right) \in H$

- if $\tau(x, y)=t$ and $\tau(x, y+1)=t^{\prime}$, then $\left(t, t^{\prime}\right) \in V$

Such a mapping $\tau$ is called a solution for $\mathcal{D}$.

For reducing this domino problem to satisfiability in region models based on $\mathcal{S}$, we fix an enumeration of all the tile positions in the first quadrant of the plane as indicated in Figure 4. The function $\lambda$ takes positive integers to $\mathbb{N} \times \mathbb{N}$-positions, i.e. $\lambda(1)=(0,0)$, $\lambda(2)=(1,0), \lambda(3)=(1,1)$, etc.

The idea of the reduction is to construct a formula $\varphi_{\mathcal{D}}$ that enforces the existence of a sequence of regions $r_{1}, r_{2}, \ldots$ such that $r_{i}$ ntpp $r_{j}$ if $i<j$. Intuitively, each region $r_{i}$ corresponds to the position $\lambda(i)$ of the first quadrant of the plane. We introduce additional regions "connecting" each $r_{i}$ with $r_{i+1}$ to facilitate writing formulas that express statements such as "if the current region $r_{i}$ satisfies $\varphi$, then the next region $r_{i+1}$ satisfies $\psi$ ", and likewise for the previous region. Similarly, we introduce additional regions that connect each region $r_{i}$ with the region $r_{j}$ such that the position $\lambda(j)$ is to the right of the position $\lambda(i)$ in the first quadrant of the plane. These latter regions allow statements such as "if the current region $r_{i}$ satisfies $\varphi$, then the region representing the position to its right satisfies $\psi "$. Using such statements, it is obviously easy to enforce the horizontal tiling condition. By virtue of our enumeration of plane positions, reaching the position above the current one is simply a matter of going to the right and then advancing by one in the enumeration. Thus, we 

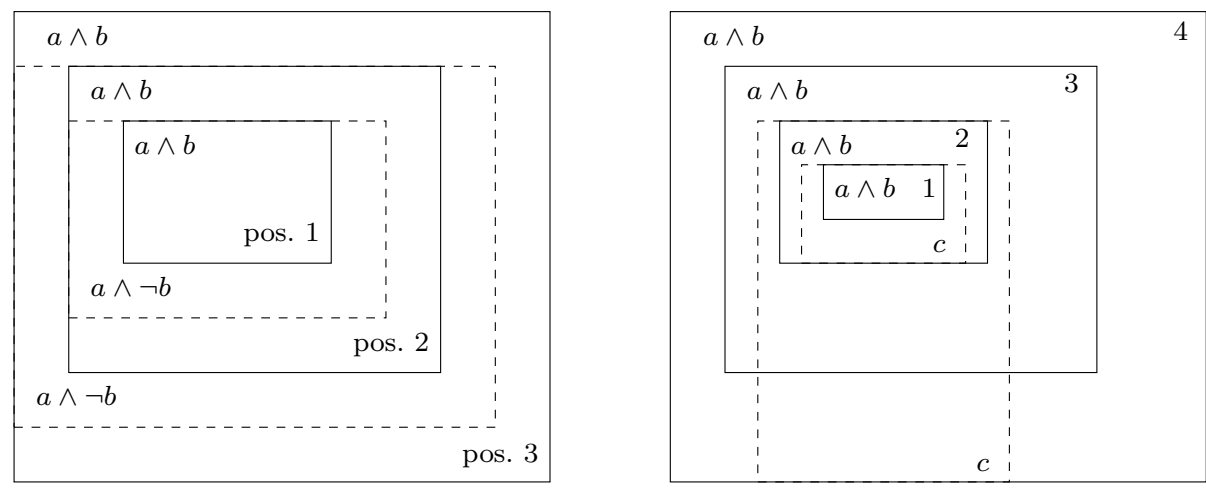

Figure 5: Left: a discrete ordering in the plane; Right: the "going right" regions.

can also enforce the vertical tiling condition. One of the main difficulties of the proof will be to enforce the existence of the connecting regions for "going to the right". The pursued solution is inspired by MR99, RZ01.

Now let $\mathcal{D}=(T, H, V)$ be a domino system. For constructing $\varphi_{\mathcal{D}}$, we use the following variables:

- for each tile type $t \in T$, a variable $p_{t}$;

- variables $a, b$, and $c$ that are used to mark important regions;

- variables wall and floor that are used to identify regions corresponding to positions from the sets $\{0\} \times \mathbb{N}$ (the wall) and $\mathbb{N} \times\{0\}$ (the floor), respectively.

The reduction formula $\varphi_{\mathcal{D}}$ is defined as

$$
a \wedge b \wedge \text { wall } \wedge \text { floor } \wedge \text { [ntppi }] \neg a \wedge \square_{u} \chi,
$$

where $\chi$ is the conjunction of a number of formulas. We list these formulas together with some intuitive explanations:

(1) Ensure that the regions $\{s \in W \mid \mathfrak{M}, s \models a\}$ are ordered by the relation pp (i.e. the union of tpp and ntpp):

$$
a \rightarrow([\mathrm{dc}] \neg a \wedge[\mathrm{ec}] \neg a \wedge[\mathrm{po}] \neg a)
$$

(2) Enforce that the regions $\{s \mid \mathfrak{M}, s \models a \wedge b\}$ are discretely ordered by ntpp. These regions will constitute the sequence $r_{1}, r_{2}, \ldots$ described above. In order to ensure discreteness, we use a sequence of alternating $a \wedge b$ and $a \wedge \neg b$ regions as shown in the left part of Figure 5

$$
\begin{aligned}
a \wedge b & \rightarrow\langle\operatorname{tpp}\rangle(a \wedge \neg b) \\
a \wedge \neg b & \rightarrow\langle\operatorname{tpp}\rangle(a \wedge b) \\
a \wedge \neg b & \rightarrow[\operatorname{tpp}](a \rightarrow b) \\
a \wedge b & \rightarrow[\operatorname{tpp}](a \rightarrow \neg b)
\end{aligned}
$$

A formal proof that these formulas work as described is given below (Point 5 of Claim 1). If we are at an $a \wedge b$ region, we can access the region corresponding to the next position in the plane (w.r.t. the fixed enumeration) and to the previous 
position using

$$
\begin{aligned}
& \diamond^{+} \varphi=\langle\operatorname{tpp}\rangle(a \wedge \neg b \wedge\langle\operatorname{tpp}\rangle(a \wedge b \wedge \varphi)) \\
& \diamond^{-} \varphi=\langle\text { tppi }\rangle(a \wedge \neg b \wedge\langle\text { tppi }\rangle(a \wedge b \wedge \varphi)) .
\end{aligned}
$$

(3) The additional regions that will eventually allow us to "go right" in the plane satisfy the propositional letter $c$ and are related to the regions corresponding to plane positions as indicated in the right part of Figure 5. For example, Position 2 in the figure is right of Position 1, and Position 4 is right of Position 2. We start with stating the following:

$$
\begin{aligned}
a \wedge b & \rightarrow\langle\operatorname{tpp}\rangle c \\
c & \rightarrow\langle\operatorname{tpp}\rangle(a \wedge b) \\
c & \rightarrow([\mathrm{dc}] \neg c \wedge[\mathrm{ec}] \neg c \wedge[\mathrm{po}] \neg c \wedge[\mathrm{tpp}] \neg c \wedge[\mathrm{tppi}] \neg c)
\end{aligned}
$$

These formulas do not yet ensure that the $c$ regions actually bring us to the correct position. Roughly spoken, they only help to ensure that "going to the right via regions satisfying $c$ " is a well-defined, monotone, and injective total function.

After further constraining the $c$ regions, we will be able to go to the right and upper position with

$$
\begin{aligned}
& \diamond^{R} \varphi=\langle\operatorname{tpp}\rangle(c \wedge\langle\operatorname{tpp}\rangle(a \wedge b \wedge \varphi)) \\
& \diamond^{U} \varphi=\diamond^{R} \diamond^{+} \varphi .
\end{aligned}
$$

Similarly, we will be able to go to the left and down:

$$
\begin{aligned}
& \diamond^{L} \varphi=\langle\text { tppi }\rangle(c \wedge\langle\text { tppi }\rangle(a \wedge b \wedge \varphi)) \\
& \diamond^{D} \varphi=\diamond^{L} \diamond^{-} \varphi .
\end{aligned}
$$

(4) Axiomatizing the behavior of tiles on the floor and on the wall ensures that our "going to the right" relation actually brings us to the expected position in the first quadrant of the plane:

$$
\begin{aligned}
(\text { floor } \wedge \text { wall }) & \rightarrow[\text { ntppi }] \neg a \\
\text { wall } & \rightarrow \diamond^{+} \text {floor } \\
\text { wall } & \rightarrow \diamond^{U} \text { wall } \\
\text { [ntppi] } \neg a \vee(\text { wall } & \left.\rightarrow \diamond^{D} \text { wall }\right) \\
a \wedge b & \rightarrow \diamond^{R} \neg \text { wall } \\
(a \wedge b \wedge \neg \text { wall }) & \rightarrow \diamond^{L} \top
\end{aligned}
$$

(5) Finally, we enforce the tiling:

$$
\begin{array}{r}
\bigwedge_{t, t^{\prime} \in T} \neg\left(p_{t} \wedge p_{t^{\prime}}\right) \\
a \wedge b \rightarrow \bigvee_{\left(t, t^{\prime}\right) \in H}\left(p_{t} \wedge \diamond^{R} p_{t^{\prime}}\right) \\
a \wedge b \rightarrow \bigvee_{\left(t, t^{\prime}\right) \in V}\left(p_{t} \wedge \diamond^{U} p_{t^{\prime}}\right)
\end{array}
$$


We now prove two lemmas asserting the correctness of the reduction. The first one is concerned with constructing solutions for $\mathcal{D}$ from region models for $\varphi_{\mathcal{D}}$. Observe that this lemma does not assume anything about the involved region model.

Lemma 8. If the formula $\varphi_{\mathcal{D}}$ is satisfiable, then the domino system $\mathcal{D}$ has a solution.

Proof Let $\mathfrak{M}=\left\langle\mathfrak{R}, p_{1}^{\mathfrak{M}}, p_{2}^{\mathfrak{M}}, \ldots\right\rangle$ be a region model of $\varphi_{\mathcal{D}}$ with $\mathfrak{R}=\langle W$, dc, ec, $\ldots\rangle$.

Claim 1. There exists a sequence $r_{1}, r_{2}, \ldots \in W$ such that

(1) $\mathfrak{M}, r_{1} \models \varphi_{\mathcal{D}}$,

(2) $r_{1}$ ntpp $r_{2}$ ntpp $r_{3}$ ntpp $\cdots$,

(3) $\mathfrak{M}, r_{i} \models a \wedge b$ for $i \geq 1$.

(4) for each $i \geq 1$, there exists a region $s_{i} \in W$ such that

(a) $r_{i} \operatorname{tpp} s_{i}$

(b) $\mathfrak{M}, s_{i} \models a \wedge \neg b$,

(c) $s_{i} \operatorname{tpp} r_{i+1}$,

(d) for each region $s$ with $r_{i} \operatorname{tpp} s$ and $\mathfrak{M}, s \models a \wedge \neg b$, we have $s=s_{i}$, and

(e) for each region $r$ with $s_{i} \operatorname{tpp} r$ and $\mathfrak{M}, r \models a \wedge b$, we have $r=r_{i+1}$,

(5) for all $r \in W$ with $\mathfrak{M}, r \models a \wedge b$, we have that $r=r_{i}$ for some $i \geq 1$ or $r_{i}$ ntpp $r$ for all $i \geq 1$.

Proof: We start with inductively constructing a sequence $r_{1}, r_{2}, \cdots \in W$ satisfying Properties 1 to 4 . Afterwards, we prove that Property 5 is also satisfied. Since $\mathfrak{M}$ is a model of $\varphi_{\mathcal{D}}$, there is a region $r_{1}$ such that $\mathfrak{M}, r_{1} \models \varphi_{\mathcal{D}}$. By definition of $\varphi_{\mathcal{D}}$, Point 3 is satisfied. Due to Formulas (5.2) and (5.3), there are regions $s_{1}$ and $r_{2}$ such that $r_{1}$ tpp $s_{1}, \mathfrak{M}, s_{1} \models a \wedge \neg b$, $s_{1}$ tpp $r_{2}$, and $\mathfrak{M}, r_{2} \models a \wedge b$. We show that all necessary Properties are satisfied:

- Point 2. Since $r_{1}$ tpp $s_{1}$ and $s_{1}$ tpp $r_{2}$, we have $r_{1}$ tpp $r_{2}$ or $r_{1}$ ntpp $r_{2}$ according to the composition table which applies to all region structures by Theorem 1 But then, the first possibility is ruled out by Formula (5.5).

- Point 4d. Suppose there is an $s \neq s_{1}$ with $r_{1}$ tpp $s$ and $\mathfrak{M}, s \models a \wedge \neg b$. Since $r_{1} \operatorname{tpp} s_{1}, s_{1}$ and $s$ are related via one of po, tpp, and tppi by the composition table. But then, the first option is ruled out by Formula (5.1) and the last two by Formula (5.4).

- Point 4e. Analogous to the previous case.

The induction step is similar: as $\mathfrak{M}, r_{i} \models a \wedge b$, we may use Formulas (5.2) and (5.3) to find the region $r_{i+1}$, and then show in the same way as above that it satisfies all relevant properties. It thus remains to prove Point 5. Assume that there is a region $r$ such that $\mathfrak{M}, r \models a \wedge b, r \neq r_{i}$ for all $i \geq 1$, and $r_{k}$ ntpp $r$ does not hold for some $k \geq 1$. Since $r_{k}$ ntpp $r$ does not hold and $r_{k} \neq r, r_{k}$ and $r$ are related by one of dc, ec, po, tpp, tppi, and ntppi. The first three possibilities are ruled out by Formula (5.1), and tpp and tppi are ruled out by Formula (5.5). It thus remains to treat the case $r_{k}$ ntppi $r$. Consider the relationship between $r_{1}$ and $r$. Since $r_{1} \neq r$ and due to Formulas (5.1) and (5.5), there are only two possibilities for this relation;

- $r$ ntpp $r_{1}$. Impossible by $\varphi_{\mathcal{D}}$ 's subformula [ntppi] $\neg a$.

- $r_{1}$ ntpp $r$. Then we have $r_{1}$ ntpp $r$ ntpp $r_{k}$. Take the maximal $i$ such that $r_{i}$ ntpp $r$ and the minimal $j$ such that $r$ ntpp $r_{j}$. Since $r \neq r_{n}$ for all $n \geq 1$, we have $j=i+1$. By Point 4 , there is a region $s$ with $r_{i}$ tpp $s, \mathfrak{M}, s \models a \wedge \neg b$, and $s$ tpp $r_{j}$. Then we have $r$ nttpi $r_{i} \operatorname{tpp} s$. By the composition table, $r$ is related to $s$ by po, tppi, or 
ntppi. On the other hand, $r$ nttp $r_{j}$ tppi $s$. By the composition table, we have one of the relations dc, ec, po, tpp, or ntpp between $r$ and $s$. Together we obtain $r$ po $s$ which contradicts Formula (5.1).

The next claim identifies the regions needed for "going right" in the plane.

Claim 2. For each $i \geq 1$, there exist regions $t_{i}$ and $u_{i}$ such that

(1) $r_{i} \operatorname{tpp} t_{i}$

(2) $\mathfrak{M}, t_{i} \models c$,

(3) for each region $t$ with $r_{i} \operatorname{tpp} t$ and $\mathfrak{M}, t \models c$, we have $t=t_{i}$,

(4) $t_{i}$ tpp $u_{i}$

(5) $\mathfrak{M}, u_{i} \models a \wedge b$,

(6) for each region $u$ with $t_{i}$ tpp $u$ and $\mathfrak{M}, u \models a \wedge b$, we have $u=u_{i}$.

Proof: Let $i \geq 1$. By Formula (5.6), there is a $t_{i}$ with $r_{i} \operatorname{tpp} t_{i}$ and $\mathfrak{M}, t_{i} \models c$. Let us show that $t_{i}$ satisfies Property 3. To this end, let $t \neq t_{i}$ such that $r_{i} \operatorname{tpp} t$ and $\mathfrak{M}, t \models c$. Then $t$ and $t_{i}$ are related via one of po, tpp, and tppi. But then, all these options are ruled out by Formula (5.8). Now for Points 4 to 6. By Formula (5.7), there is an $r$ such that $t_{i} \operatorname{tpp} r$ and $\mathfrak{M}, r \models a \wedge b$. Point 6 can now be be proved analogously to Point 3, using Formulas (5.1) and (5.5) instead of Formula (5.8). This finishes the proof of Claim 2.

The next claim states that the regions $u_{i}$ fixed in Claim 2 are ordered by ntpp.

Claim 3. Let $i, j \geq 1$ with $i<j$. Then $u_{i}$ ntpp $u_{j}$.

Proof: By Claims 1 and 2, we have (i) $r_{i}$ ntpp $r_{j}$, (ii) $r_{i} \operatorname{tpp} t_{i}$, and (iii) $r_{j} \operatorname{tpp} t_{j}$. By the composition table, (i) and (iii) yield $r_{i}$ ntpp $t_{j}$, which together with (ii) implies that $t_{i}$ and $t_{j}$ are related by po, tpp, or ntpp. Since $\mathfrak{M}, t_{i} \models c$ and $\mathfrak{M}, t_{j} \models c$ by Claim 2 , all but the last possibility are ruled out by Formula (5.8). Therefore $t_{i}$ ntpp $t_{j}$ which together with $t_{j} \operatorname{tpp} u_{j}$ (Claim 2) implies $t_{i}$ ntpp $u_{j}$. By Claim 2 we also have $t_{i} \operatorname{tpp} u_{i}$ which by the composition table implies that $u_{i}$ and $u_{j}$ are related by po, tpp, or ntpp. Again by Claim 2, $\mathfrak{M}, u_{i} \models a \wedge b$ and $\mathfrak{M}, u_{j} \models a \wedge b$. Hence the first two possibilities are ruled out by Formulas (5.1) and (5.5). It follows that $u_{i} \operatorname{ntpp} u_{j}$, as required.

Before proceeding, let us introduce some notation.

- for $i, j>0$, we write $i \Rightarrow j$ if the tile position $\lambda(j)$ can be reached from $\lambda(i)$ by going one step to the right. Similarly, we define a relation $i \Uparrow j$ for going one step up;

- for $i, j>0$ we write $r_{i} \rightarrow r_{j}$ if $u_{i}=r_{j}$. Similarly, we write $r_{i} \uparrow r_{j}$ if $r_{i} \rightarrow r_{j-1}$.

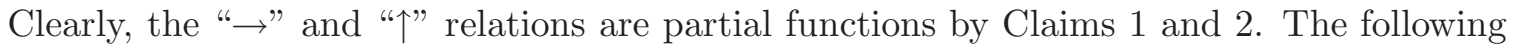
claim establishes some other important properties of " $\rightarrow$ ": first, it moves only ahead in the sequence $r_{1}, r_{2}, \ldots$, but never back. And second, it is monotone and injective.

Claim 4. Let $i, j \geq 1$. Then the following holds:

(1) if $r_{i} \rightarrow r_{j}$, then $i<j$;

(2) if $i<j, r_{i} \rightarrow r_{k}$, and $r_{j} \rightarrow r_{\ell}$, then $k<\ell$;

Proof: First for Point 1. Suppose $r_{i} \rightarrow r_{j}$ and $i \geq j$. Then $u_{i}=r_{j}$ and, by Claim 2, $r_{i}$ tpp $t_{i}$ tpp $r_{j}$. By the composition table, $r_{i}$ is related to $r_{j}$ by tpp or ntpp. But by Claim $1, i \geq j$ implies $r_{i}$ eq $r_{j}$ or $r_{i}$ ntppi $r_{j}$. We have derived a contradiction. Hence $r_{i} \rightarrow r_{j}$ implies $i<j$.

Now for Point 2. Assume $i<j, r_{i} \rightarrow r_{k}$, and $r_{j} \rightarrow r_{\ell}$. We have $u_{i}=r_{k}$ and $u_{j}=r_{\ell}$. Hence, by Claim $3, r_{k}$ ntpp $r_{\ell}$. Using Claim 1 and the composition table, we derive $k<\ell$. 
The following claim establishes the core part of the proof: the fact that the " $\rightarrow$ " relation "coincides" with the " $\Rightarrow$ " relation, and similar for " $\uparrow "$ and " $\uparrow "$. More precisely, this follows from Point 3 of the following claim. For technical reasons, we simultaneously prove some other, technical properties. The proof of this claim follows the lines of Marx and Reynolds MR99.

Claim 5. Let $i \geq 1$ and $i \Rightarrow j$. Then the following holds:

(1) if $\lambda(j)$ is on the floor, then $\mathfrak{M}, r_{j} \models$ floor;

(2) $\mathfrak{M}, r_{j} \not \neq$ wall;

(3) $r_{i} \rightarrow r_{j}$ and $r_{i} \uparrow r_{j+1}$.

(4) if $\lambda(j+1)$ is on the wall, then $\mathfrak{M}, r_{j+1} \models$ wall

Proof: All subclaims are proved simultaneously by induction on $i$. First for the induction start. Then we have $i=1$ and $j=2$.

(1) Clearly, $\lambda(2)$ is on the floor. Since $\mathfrak{M}, r_{1} \models \varphi_{\mathcal{D}}$, we have $\mathfrak{M}, r_{1} \models$ wall. Thus Formula (5.10) yields $\mathfrak{M}, r_{2} \models$ floor.

(2) We have $1 \Rightarrow 2$. Point 1 gives us $\mathfrak{M}, r_{2} \models$ floor. Since $r_{1}$ ntpp $r_{2}$, we also have $\mathfrak{M}, r_{2} \not \forall\left[\right.$ ntppi] $\neg a$. Thus, Formula (5.9) yields $\mathfrak{M}, r_{2} \not \models$ wall.

(3) By Point 2, we have $\mathfrak{M}, r_{2} \not \models$ wall. By Formula (5.14), there are regions $r, s \in W$ such that $\mathfrak{M}, r \models a \wedge b, r \operatorname{tpp} s, \mathfrak{M}, s \models c$, and $s$ tpp $r_{2}$. By Point 5 of Claim 1, we have either $r=r_{i}$ for some $i \geq 1$ or $r_{i}$ ntpp $r$ for all $i \geq 1$. In the first case, we have $r_{i} \rightarrow r_{2}$. Claim 4.1 yields $i=1$ and we are done. In the second case, we have $r_{2}$ ntpp $r$ : contradiction to $r$ tpp $s$ and $s$ tpp $r_{2}$. Finally, $r_{1} \uparrow r_{3}$ is an immediate consequence of $r_{1} \rightarrow r_{2}$ and the definition of " $\uparrow$ ".

(4) Since $\lambda(3)$ is on the wall, we have to show that $\mathfrak{M}, r_{3} \models$ wall. By Point 3 , we have $r_{1} \uparrow r_{3}$. Thus, Formula (5.11) yields the desired result.

Now for the induction step.

(1) Suppose that $\lambda(j)$ is on the floor. Since obviously $j>1, \lambda(j-1)$ is on the wall. Since $i>1$, there is a $k$ with $i-1 \Rightarrow k$. It is readily checked that $j-1=k+1$. Thus, IH (Point 4 ) yields $\mathfrak{M}, r_{j-1} \models$ wall and we can use Formula (5.10) to conclude that $\mathfrak{M}, r_{j} \models$ floor as required.

(2) First assume that $\lambda(j)$ is on the floor. Since $j>1$, we have $\mathfrak{M}, r_{j} \forall \forall[$ ntppi] $\neg a$. Thus, Point 1 and Formula (5.9) yield $\mathfrak{M}, r_{j} \not \models$ wall as required.

Now assume that $\lambda(j)$ is not on the floor. Suppose, to the contrary of what is to be shown, that $\mathfrak{M}, r_{j} \models$ wall. Since $j>1$, we have $\mathfrak{M}, r_{j} \forall \forall[$ ntppi] $\neg a$. Thus, by Formula (5.12) we obtain $\mathfrak{M}, r_{j} \models \diamond^{D}$ wall. Since $j$ is not on the floor, $i \Rightarrow j$ implies $i-1 \Rightarrow j-1$. Thus, the IH (Point 3) yields $r_{i-1} \uparrow r_{j}$. Hence, we can use $\mathfrak{M}, r_{j} \models \diamond^{D}$ wall to derive $\mathfrak{M}, r_{i-1} \models$ wall. By IH (Point 2 ), we cannot have $m \Rightarrow i-1$ for any $m$. Thus, $\lambda(i-1)$ is on the wall implying that $\lambda(i)$ is on the floor. We have established a contradiction since, with $i \Rightarrow j$, this yields that $j$ is on the floor.

(3) We start with showing $r_{i} \rightarrow r_{j}$. To this end, let us prove that we have $r_{k} \rightarrow r_{j}$ for some $k<j$. By Point 2, we have $\mathfrak{M}, r_{j} \not \models$ wall. By Formula (5.14), there are regions $r, s \in W$ such that $\mathfrak{M}, r \models a \wedge b, r$ tpp $s, \mathfrak{M}, s \models c$, and $s$ tpp $r_{j}$. By Point 5 of Claim 1, we have either $r=r_{k}$ for some $k \geq 1$ or $r_{n}$ ntpp $r$ for all $n \geq 1$. In the first case, Claim 4.1 yields $k<j$ and we are done. In the second case, we have $r_{j}$ ntpp $r$ : contradiction to $r$ tpp $s$ and $s$ tpp $r_{j}$. 
Next, we show that $k=i$. To this end, assume that $k \neq i$. We distinguish two cases:

- $k<i$. Let $\ell$ be such that $k \Rightarrow \ell$. By IH (Point 3), we have $r_{k} \rightarrow r_{\ell}$. Due to functionality of " $\rightarrow$ " (Claim 2) and since $r_{k} \rightarrow r_{j}$, we have $\ell=j$. Due to the injectivity of " $\Rightarrow$ ", we get $k=i$, which is a contradiction.

- $i<k$. By Claim 2, we have $r_{i}$ ntpp $u_{i}$ and $\mathfrak{M}, u_{i} \models a \wedge b$. By Point 5 of Claim 1, we have either (i) $u_{i}=r_{\ell}$ for some $\ell \geq 1$ or (ii) $r_{n}$ ntpp $u_{i}$ for all $n \geq 1$. In Case (ii), in particular we have $r_{j}$ ntpp $u_{i}$. Since $r_{k} \rightarrow r_{j}$, we have $r_{j}=u_{k}$, and thus $u_{k}$ ntpp $u_{i}$. As $i<k$, we have obtained a contradiction to Claim 3. Thus, Case (ii) is impossible and we conclude $u_{i}=r_{\ell}$ for some $\ell \geq 1$. Next, we make a case distinction as follows:

$-\ell<j$. There are two subcases: the tile position $\lambda(\ell)$ may or may not be on the wall.

First assume that it is not. Then there is an $h<\ell$ with $h \Rightarrow \ell$. By definition of the " $\Rightarrow$ " function, $i \Rightarrow j, h \Rightarrow \ell$, and $\ell<j$ this implies $h<i$. Thus we can use IH (Point 3) to conclude $r_{h} \rightarrow r_{\ell}$, a contradiction to the injectivity of " $\rightarrow$ " (Claim 4.2) and the facts that $r_{i} \rightarrow r_{\ell}$ and $h<i$. Now assume that $\lambda(\ell)$ is on the wall. Since $1<i<\ell$, there is a $h$ such that $h \uparrow \ell$ and $h \rightarrow \ell-1$. Thus, IH (Point 4) yields $\mathfrak{M}, r_{\ell} \models$ wall. But then, $r_{i} \rightarrow r_{\ell}$ and Formula (5.13) yield a contradiction.

$-\ell=j$. Then $r_{i} \rightarrow r_{j}$ and $r_{k} \rightarrow r_{j}$, which is a contradiction to the injectivity of " $\rightarrow$ " (Claim 4.2) since $i \neq k$.

$-\ell>j$. Contradiction to the monotonicity of " $\rightarrow$ " (Claim 4.2).

The second part of Point 3, i.e. $r_{i} \uparrow r_{j+1}$, is now an immediate consequence of the fact that $r_{i} \rightarrow r_{j}$ and the definition of " $\uparrow "$.

(4) Suppose that $\lambda(j+1)$ is on the wall. Then $\lambda(i)$ is also on the wall. Since additionally $i>1$, there is a $k$ such that $k \Uparrow i$ and $k \Rightarrow i-1$. By IH (Point 4), the latter yields $\mathfrak{M}, r_{i} \models$ wall. Since Point 3 yields $r_{i} \uparrow r_{j+1}$, Formula (5.11) yields $\mathfrak{M}, r_{j+1} \models$ wall.

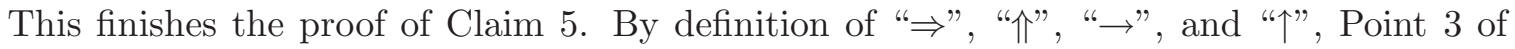
this claim yields the following:

$$
i \Rightarrow j \text { implies } r_{i} \rightarrow r_{j} \quad \text { and } \quad i \Uparrow j \text { implies } r_{i} \uparrow r_{j} .
$$

Using this property, we can finally define the solution of $\mathcal{D}$ : set $\tau(i, j)$ to the unique $t \in T$ such that $\mathfrak{M}, r_{n} \models p_{t}$, where $\lambda(n)=(i, j)$. This is well-defined due to Formulas (5.15) and (5.16). Thus, it remains to check the matching conditions:

- Let $(i, j) \in \mathbb{N}^{2}, \lambda(n)=(i, j)$, and $\lambda(m)=(i+1, j)$. Then $n \Rightarrow m$. By $(*)$, this yields $r_{n} \rightarrow r_{m}$. By Formula (5.16), there are $\left(t, t^{\prime}\right) \in H$ such that $\mathfrak{M}, r_{n} \models p_{t}$ and $\mathfrak{M}, r_{m} \models p_{t^{\prime}}$. Since this implies $\tau(i, j)=t$ and $\tau(i+1, j)=t^{\prime}$, the horizontal matching condition is satisfied.

- The vertical matching condition can be verified analogously using Formula (5.17).

The second lemma deals with the construction of models for $\varphi_{\mathcal{D}}$ from solutions for $\mathcal{D}$. Here, we have to make a suitable assumption on the class of region structures $\mathcal{S}$ for the construction to succeed. One possible such assumption is given in Theorem 5 It turns out, however, that the following more general condition is also sufficient. 
Definition 9 (Domino ready). Let $\mathfrak{R}=\langle W, \mathrm{dc}, \mathrm{ec}, \ldots\rangle$ be a region structure. Then $\mathfrak{R}$ is called domino ready if it satisfies the following property: the set $W$ contains sequences $x_{1}, x_{2}, \ldots$ and $y_{1}, y_{2}, \ldots$ such that, for $i, j \geq 1$, we have

(1) $x_{i} \operatorname{tpp} x_{i+1}$;

(2) $x_{i}$ ntpp $x_{j}$ if $j>i+1$;

(3) $x_{2 i-1}$ tpp $y_{i}$;

(4) $y_{i}$ tpp $x_{2 j-1}$ iff the position $\lambda(j)$ can be reached from $\lambda(i)$ by going one step to the right;

(5) $y_{i} \operatorname{ntpp} y_{j}$ if $j>i$.

Before discussing this property in some more detail, let us show that it is indeed suitable for our proof.

Lemma 10. Let $\mathfrak{R}=\langle W, \mathrm{dc}, \mathrm{ec}, \ldots\rangle$ be a region structure that is domino ready. If the domino system $\mathcal{D}$ has a solution, then the formula $\varphi_{\mathcal{D}}$ is satisfiable in a region model based on $\mathfrak{R}$.

Proof Let $\mathfrak{R}$ be a region structure that is domino ready, $\mathcal{D}=(T, H, V)$ a domino system, and $\tau$ a solution of $\mathcal{D}$. We introduce new names for the regions listed in Definition 9 that are closer to the names used in the proof of Lemma 8

- $r_{i}:=x_{2 i-1}$ for $i \geq 1$;

- $s_{i}:=x_{2 i}$ for $i \geq 1$;

- $t_{i}:=y_{i}$.

Now define a region model $\mathfrak{M}$ based on $\mathfrak{R}$ by interpreting the propositional letters as follows:

- $a^{\mathfrak{M}}=\left\{r_{i}, s_{i} \mid i \geq 1\right\}$;

- $b^{\mathfrak{M}}=\left\{r_{i} \mid i \geq 1\right\}$

- $c^{\mathfrak{M}}=\left\{t_{i} \mid i \geq 1\right\}$

- wall $^{\mathfrak{M}}=\left\{r_{i} \mid \lambda(i)\right.$ is on the wall $\}$

- floor $^{\mathfrak{M}}=\left\{r_{i} \mid \lambda(i)\right.$ is on the floor $\}$;

- $p_{t}^{\mathfrak{M}}=\left\{r_{i} \mid \tau(\lambda(i))=t\right\}$.

It is now easy to verify that $\chi$ is satisfied by every region of $\mathfrak{M}$, and that $\mathfrak{M}, r_{1} \models \varphi_{\mathcal{D}}$.

We have thus proved the following theorem.

Theorem 11. Let $\mathcal{S} \subseteq \mathcal{R S}$ such that some $\mathfrak{R} \in \mathcal{S}$ is domino ready. Then $L_{\mathrm{RCC} 8}(\mathcal{S})$ is undecidable.

We now show that this theorem implies Theorem 5

Lemma 12. Each region structure $\mathfrak{R}\left(\mathbb{R}^{n}, U\right)$ with $n>0$ and $\mathbb{R}_{\text {rect }}^{n} \subseteq U$ is domino ready.

Proof We start with $n=1$. Thus, we must exhibit the existence of two sequences of convex, closed intervals $x_{1}, x_{2}, \ldots$ and $y_{1}, y_{2}, \ldots$ satisfying Properties 1 to 5 from Definition 9 for $i \geq 1$, set

- $x_{i}:=[-j, j]$ if $i=2 j-1$;

- $x_{i}:=[-j, j+1]$ if $i=2 j$;

- $y_{i}:=[-i, j]$ if $\lambda(j)$ is the position reached from $\lambda(i)$ by going a single step to the right.

It is readily checked that these sequences of intervals are as required. To find sequences for $n>1$, just use the $n$-dimensional products of these intervals. 
Note that we can also prove this lemma if we admit only bounded rectangles of $\mathbb{R}^{n}$ as regions: the construction from Lemma 12 can easily be modified so that the sequence of $a \wedge b$-rectangles converges against a finite rectangle, rather than against $\mathbb{R}^{n}$.

indeed more general than Theorem 5. For example, region structures that are obtained by choosing all closed circles or ellipses as regions are easily seen to be domino ready, but they do not satisfy the condition from Theorem 5

\section{Recursive Enumerability}

In this section, we discuss the question whether modal logics of topological relations are recursively enumerable. We start with a simple observation.

Theorem 13. For $n>0, L_{\mathrm{RCC} 8}(\mathcal{R S})=L_{\mathrm{RCC} 8}^{\mathrm{S}}(\mathcal{T O P})=L_{\mathrm{RCC} 8}^{\mathrm{S}}\left(\mathbb{R}^{n}, \mathbb{R}_{\text {reg }}^{n}\right)$ are recursively enumerable.

Proof The equality has already been shown in Theorem $4 L_{\mathrm{RCC} 8}(\mathcal{R S})$ is recursively enumerable since (i) the class of all region structures $\mathcal{R} S$ is first-order definable (c.f. its definition in Section 21); (ii) it is a standard result that $\mathcal{L}_{\mathrm{RCC} 8}$ formulas can be translated into equivalent formulas of $\mathcal{F} \mathcal{O}_{\mathrm{RCC} 8}^{m}$ (see Section 4 ); (iii) first-order logic is recursively enumerable.

An alternative proof of Theorem 13 can be obtained by explicitly giving an axiomatization of $L_{\mathrm{RCC} 8}(\mathcal{R S})$. Since this is interesting in its own right, in the following we develop such an axiomatization based on a non-standard rule. Non-standard rules, which are sometimes called non-orthodox or Gabbay-Burgess style rules, were introduced in temporal logic in Bur80, Gab81a and often enable finite axiomatizations of modal logics for which no finite standard axiomatization (using only the rules modus ponens and necessitation) is known. For $L_{\mathrm{RCC} 8}(\mathcal{R S})$, we leave it as an open problem whether a finite standard axiomatization exists. To guarantee a simple presentation, we develop an axiomatization for the extension of our language $\mathcal{L}_{\mathrm{RCC} 8}$ with countably many nominals, i.e. a new sort of variables $i, j, k, \ldots$ interpreted in singleton sets. As noted in Section 3, nominals can be defined in the original language, but here it is more convenient to treat them as first-class citizens since this enables the application of general completeness results from modal logic. ${ }^{3}$ The universal box $\square_{u}$ is still used as an abbreviation. Then the logic of all region structures is axiomatized by the following axiom and rule schemata, where $\varphi$ and $\psi$ range over formulas of $\mathcal{L}_{\mathrm{RCC} 8}$ extended with nominals, $i$ over the nominals, and $r, r_{1}, r_{2}$ over the RCC8-relations:

- axioms of propositional logic;

- $[\mathrm{r}](\varphi \rightarrow \psi) \rightarrow([\mathrm{r}] \varphi \rightarrow[\mathrm{r}] \psi)$

- $\left\langle\mathrm{r}_{1}\right\rangle i \rightarrow \neg\left\langle\mathrm{r}_{2}\right\rangle i$, for $\mathrm{r}_{1} \neq \mathrm{r}_{2}$. These axioms ensure that the $\mathrm{r}$ are mutually disjoint;

- $\left\langle r_{1}\right\rangle\left\langle r_{2}\right\rangle \varphi \rightarrow\left\langle q_{1}\right\rangle \varphi \vee \cdots \vee\left\langle q_{k}\right\rangle \varphi$, whenever

$$
\forall x \forall y \forall z\left(\left(\mathrm{r}_{1}(x, y) \wedge \mathrm{r}_{2}(y, z)\right) \rightarrow\left(\mathrm{q}_{1}(x, z) \vee \cdots \vee \mathrm{q}_{k}(x, z)\right)\right.
$$

is in the RCC8-composition table;

- $\varphi \rightarrow[r]\langle r\rangle \varphi$, whenever $r$ is symmetric;

- $\varphi \rightarrow\left[r_{1}\right]\left\langle r_{2}\right\rangle \varphi$ and $\varphi \rightarrow\left[r_{2}\right]\left\langle r_{1}\right\rangle \varphi$, whenever $r_{1}$ is the inverse of $r_{2}$;

\footnotetext{
${ }^{3}$ One could also give a finite non-standard axiomatization without adding nominals to the language by making use of the definable difference modality $\square_{d}$ and then applying a general completeness result of Ven92] (Theorem 2.7.7).
} 
- $\square_{u} \varphi \rightarrow \varphi, \square_{u} \varphi \rightarrow \square_{u} \square_{u} \varphi$, and $\varphi \rightarrow \square_{u} \diamond_{u} \varphi$. These axioms ensure that $\square_{u}$ is a S5-modality;

- $[\mathrm{eq}] \varphi \leftrightarrow \varphi$;

- $\diamond_{u} i$. This axiom ensures that the interpretation of nominals is non-empty;

- $\diamond_{u}(i \wedge \varphi) \rightarrow \square_{u}(i \rightarrow \varphi)$. This axiom together with the rule cov below ensures that the interpretation of nominals are at most singleton sets;

- the rules modus ponens, necessitation, and the non-standard rule cov:

$$
\frac{\varphi, \varphi \rightarrow \psi}{\psi} \quad \frac{\varphi}{\square_{u} \varphi} \quad \frac{i \rightarrow \varphi}{\varphi} \text { if } i \text { not in } \varphi
$$

It is straightfoward to prove the soundness of this axiomatization. Completeness follows from a general completeness result of GV01 for logics with nominals and the universal modality, since all the axioms not involving nominals are Sahlqvist axioms, and, for each modal operator $[r]$, we have an operator $\left[\mathrm{r}^{-1}\right]$ interpreted by the converse of the accessibility relation for $[\mathrm{r}]$.

Returning to our original proof of Theorem 13. we note that there is another class of logics for which recursive enumerability can be proved using first-order logic: $L_{\mathrm{RCC} 8}^{\mathrm{S}}\left(\mathbb{R}^{n}, \mathbb{R}_{\text {rect }}^{n}\right)$, $n \geq 1$. In this case, however, we need a different translation that takes into account the underlying region structures and the shape of regions. The proof is similar to the translation of interval temporal logic into first-order logic given by Halpern and Shoham in HS91. The important difference is that Halpern and Shoham use their translation to prove recursive enumerability of interval temporal logics determined by full interval structures that are first-order definable, whereas we prove recursive enumerability of a logic determined by substructures of a structure that is not first-order definable. The proof can be found in Appendix C

Theorem 14. For $n \geq 1, L_{\mathrm{RCC} 8}^{\mathrm{S}}\left(\mathbb{R}^{n}, \mathbb{R}_{\text {rect }}^{n}\right)$ is recursively enumerable.

With the exception of the class of logics $L_{\mathrm{RCC} 8}^{\mathrm{S}}\left(\mathbb{R}^{n}, \mathbb{R}_{\text {conv }}^{n}\right)$, whose recursive enumerability status we have to leave as an open problem, it thus turns out that all logics introduced in Section 4 that are based on substructures of concrete region structures are recursively enumerable. ${ }^{4}$ Interestingly, this is not the case for logics based on full concrete region structures, and thus going from full concrete region structures to substructures yields a computational benefit. In the following, we prove that most of the logics introduced in Section 4 based on full concrete region structures are $\Pi_{1}^{1}$-hard, and thus not recursively enumerable. Note, however, that the conditions listed in the theorem are much less general than those from Theorem [1].

Theorem 15. The following logics are $\Pi_{1}^{1}$-hard: $L_{\mathrm{RCC} 8}(\mathcal{T O P})$ and $L_{\mathrm{RCC} 8}\left(\mathbb{R}^{n}, U_{n}\right)$ with $U_{n} \in\left\{\mathbb{R}_{\text {reg }}^{n}, \mathbb{R}_{\text {conv }}^{n}\right\}$ and $n \geq 1$.

To prove Theorem [15] the domino problem of Definition 7 is modified by requiring that, in solutions, a distinguished tile $t_{0} \in T$ occurs infinitely often in the first column of the first quadrant, i.e. on the wall. It has been shown in Har85 that this variant of the domino problem is $\Sigma_{1}^{1}$-hard. Since we reduce it to satisfiability as in the proof of Theorem 5 this yields a $\Pi_{1}^{1}$-hardness bound for validity.

As a first step toward reducing this stronger variant of the domino problem, we extend $\varphi_{\mathcal{D}}$ with the following conjunct stating that $\mathfrak{M}, s \models \varphi_{\mathcal{D}}$ implies that we find an infinite sequence

\footnotetext{
${ }^{4}$ Recall that concrete region structures are those region structures induced by topological spaces.
} 
of regions $r_{1}, r_{2}, \ldots$ such that $s=r_{1}, r_{i}$ ntpp $r_{i+1}$, and $\mathfrak{M}, r_{i} \models a \wedge b \wedge$ wall $\wedge p_{t_{0}}$ for all $i \geq 1$ :

$$
\square_{u}\left(a \wedge b \rightarrow\langle\operatorname{ntpp}\rangle\left(a \wedge b \wedge \text { wall } \wedge p_{t_{0}}\right)\right)
$$

However, this is not yet sufficient: in models of $\varphi_{\mathcal{D}}$, we can have not only one discrete ordering of $a \wedge b$ regions, but rather many such orderings that are "stacked". For example, there could be two sequences of regions $r_{1}, r_{2}, \ldots$, and $r_{1}^{\prime}, r_{2}^{\prime}, \ldots$ such that

$$
r_{1} \text { ntpp } r_{2} \text { ntpp } r_{3} \cdots, \quad r_{1}^{\prime} \operatorname{ntpp} r_{2}^{\prime} \operatorname{ntpp} r_{3}^{\prime} \cdots, \quad \text { and } r_{i} \text { ntpp } r_{j}^{\prime} \text { for all } i, j \geq 1 \text {. }
$$

Due to this effect, the above formula does not enforce that the main ordering (there is only one for which we can ensure a proper "going to the right relation") has infinitely many occurrences of $t_{0}$.

The obvious solution to this problem is to prevent stacked orderings. This is done by enforcing that there is only one "limit region", i.e. only one region approached by an infinite sequence of $a$-regions in the limit. We add the following formula to $\varphi_{\mathcal{D}}$ :

$$
\square_{u}([\mathrm{tppi}]\langle\mathrm{po}\rangle a \rightarrow(\neg a \wedge[\mathrm{tpp}] \neg a \wedge[\mathrm{ntpp}] \neg a))
$$

Let $\varphi_{\mathcal{D}}^{\prime}$ be the resulting extension of $\varphi_{\mathcal{D}}$. The classes of region structures to which the extended reduction applies is more restricted than for the original one. We require that they are concrete, i.e. induced by a topological space, and additionally adopt the following property:

Definition 16 (Closed under infinite unions). Suppose that $\mathfrak{R}=\mathfrak{R}\left(\mathfrak{T}, U_{\mathfrak{T}}\right)=\langle W$, dc, ec, .. $\rangle$ is a concrete region structure. Then $\mathfrak{R}$ is closed under infinite unions if, for any sequence $r_{1}, r_{2}, \ldots \in W$ with $r_{1}$ ntpp $r_{2}$ ntpp $r_{3} \cdots$, we have $\mathbb{C} \mathbb{I}\left(\bigcup_{i \in \omega} r_{i}\right) \in W$.

We can now formulate the first part of correctness for the extended reduction.

Lemma 17. Let $\mathfrak{R}\left(\mathfrak{T}, U_{\mathfrak{T}}\right)=\langle W, \mathrm{dc}, \mathrm{ec}, \ldots\rangle$ be a concrete region structure that is closed under infinite unions. If the formula $\varphi_{\mathcal{D}}^{\prime}$ is satisfiable in a region model based on $\mathfrak{R}$, then the domino system $\mathcal{D}$ has a solution with $t_{0}$ occurring infinitely often on the wall.

Proof Let $\mathfrak{R}\left(\mathfrak{T}, U_{\mathfrak{T}}\right)=\langle W$, dc, ec, ... be a concrete region structure that is closed under infinite unions, $\mathfrak{M}=\left\langle\mathfrak{R}, p_{1}^{\mathfrak{M}}, p_{2}^{\mathfrak{M}}, \ldots\right\rangle$ a region model based on $\mathfrak{R}\left(\mathfrak{T}, U_{\mathfrak{T}}\right)$, and $w \in W$ such that $\mathfrak{M}, w \models \varphi_{\mathcal{D}}^{\prime}$. We may establish Claims 1 to 5 as in the proof of Lemma 8 , and we will use the same terminology in what follows. We first strengthen Point 5 of Claim 1:

Claim 1'. There exists a sequence $r_{1}, r_{2}, \cdots \in W$ such that

(1) $\mathfrak{M}, r_{1} \models \varphi_{\mathcal{D}}$,

(2) $r_{1}$ ntpp $r_{2}$ ntpp $r_{3}$ ntpp $\cdots$,

(3) $\mathfrak{M}, r_{i} \models a \wedge b$ for $i \geq 1$.

(4) for each $i \geq 1$, there exists a region $s_{i} \in W$ such that

(a) $r_{i} \operatorname{tpp} s_{i}$

(b) $\mathfrak{M}, s_{i} \models a \wedge \neg b$,

(c) $s_{i} \operatorname{tpp} r_{i+1}$,

(d) for each region $s$ with $r_{i} \operatorname{tpp} s$ and $\mathfrak{M}, s \models a \wedge \neg b$, we have $s=s_{i}$, and

(e) for each region $r$ with $s_{i} \operatorname{tpp} r$ and $\mathfrak{M}, r \models a \wedge b$, we have $r=r_{i+1}$,

(5') for all $r \in W$ with $\mathfrak{M}, r \models a \wedge b$, we have $r=r_{i}$ for some $i \geq 1$. 
Proof: We construct the sequence $r_{1}, r_{2}, \ldots$ as in the proof of Claim 1. Since Properties 1 to 4 are satisfied by construction, it remains to prove Point 5': as $\mathfrak{R}\left(\mathfrak{T}, U_{\mathfrak{T}}\right)$ is closed under infinite unions, we have $t=\mathbb{C} \mathbb{I}\left(\bigcup_{i \in \omega} r_{i}\right) \in W$. We first show that

$$
t \models[\text { tppi }]\langle\text { po }\rangle a
$$

To this end, suppose $t$ tppi $q$. Then we have the following:

(1) $q-r_{i} \neq \emptyset$ for all $i>0$.

Since $t$ tppi $q$, there exists $x \in q$ such that $x \notin \mathbb{I}(t)$. Suppose $x \in r_{i}$, for some $r_{i}$. Since $r_{i}$ ntpp $r_{i+1}$, this yields $x \in \mathbb{I}\left(r_{i+1}\right)$. By definition of $t$, we get $x \in \mathbb{I}(t)$ and have a contradiction.

(2) There exists $n>0$ such that $i \geq n$ implies $r_{i}-q \neq \emptyset$.

Suppose $r_{i} \subseteq q$, for all $i>0$. Then $s=\bigcup_{i \in \omega} r_{i} \subseteq q$. Since $q \in U_{\mathfrak{T}}$, we have $q=\mathbb{C I}(q)$. Thus $t=\mathbb{C I}(s) \subseteq q$, and we have a contradiction to $t$ tppi $q$.

(3) There exists $m>0$ such that $j \geq m$ implies $\mathbb{I}\left(r_{j}\right) \cap \mathbb{I}(q) \neq \emptyset$.

Since $q=\mathbb{C I}(q)$, we have $\mathbb{I}(q) \neq \emptyset$. Take any $x \in \mathbb{I}(q)$. Since $t=\mathbb{C I}\left(\bigcup_{i \in \omega} r_{i}\right)$ and $t$ tppi $q$, this yields $x \in \bigcup_{i \in \omega} r_{i}$. Thus there is a $j$ with $x \in r_{j}$. Then $x \in \mathbb{I}\left(r_{j+1}\right)$. Set $m:=j+1$. Since $r_{m}$ ntpp $r_{i}$ for all $i>m$, we have $x \in \mathbb{I}(q) \cap \mathbb{I}\left(r_{j+1}\right)$ for all $i \geq m$.

Take $k=\max \{n, m\}$. Using the above Points 1 to 3 and the definition of the po relation, it is easily verified that $q$ po $r_{k}$, thus finishing the proof of $(*)$.

Now we can establish Point 5'. By Point 5 of the original Claim 1, for all $r \in W$ with $\mathfrak{M}, r \models a \wedge b$, we have that $r=r_{i}$ for some $i \geq 1$ or $r_{i}$ ntpp $r$ for all $i \geq 1$. It thus suffices to show that the latter alternative yields a contradiction. Thus assume $r_{i}$ ntpp $r$ for all $i \geq 1$. Since $r_{1}$ ntpp $r_{2}$ ntpp $\cdots$ and $t=\mathbb{C I}\left(\bigcup_{i \in \omega} r_{i}\right)$, it is not hard to verify that this yields $r=t$, $t$ tpp $r$, or $t$ ntpp $r$. By $(*), t$ satisfies [tppi] $\langle$ po $\rangle a$. By Formula (6.2), $t$ thus also satisfies $\neg a \wedge[\mathrm{tpp}] \neg a \wedge[\mathrm{ntpp}] \neg a$ : contradiction since $\mathfrak{M}, r \models a$.

Lemma 8 By Point 5' of Claim 1' and Formula (6.1), this solution is such that the tile $t_{0}$ occurs infinitely often on the wall.

For the second part of correctness, we consider region structures $\mathfrak{R}\left(\mathbb{R}^{n}, U\right)$ with $\mathbb{R}_{\text {rect }}^{n} \subseteq$ $U$ as in Theorem [ In contrast to the previous section, it does not suffice to demand that region structures are domino ready.

Lemma 18. If the domino system $\mathcal{D}$ has a solution with $t_{0}$ occurring infinitely often on the wall, then the formula $\varphi_{\mathcal{D}}^{\prime}$ is satisfiable in a region model based on $\Re\left(\mathbb{R}^{n}, U\right)$, for each $n \geq 1$ and each $U$ with $\mathbb{R}_{\text {rect }}^{n} \subseteq U \subseteq \mathbb{R}_{\text {reg }}^{n}$.

Proof Let $\tau$ be a solution of $\mathcal{D}$ with $t_{0}$ appearing infinitely often on the wall. It was shown in the proof of Lemma 12 that the region spaces we are considering are domino ready. Thus we can use $\tau$ to construct a model $\mathfrak{M}$ based on the region space $\mathfrak{R}\left(\mathbb{R}^{n}, U\right)$ exactly as in the proof of Lemma 10, It suffices to show that $\mathfrak{M}$ satisfies, additionally, Formulas (6.1) and (6.2). This is easy for Formula (6.1) since $\tau$ has been chosen such that $t_{0}$ appears infinitely often. Thus, let us concentrate on Formula (6.2).

Let $r_{1}, r_{2}, \ldots$ be the regions from the construction of $\mathfrak{M}$ in the proof of Lemma 10] If

$$
t=\mathbb{C} \mathbb{I}\left(\bigcup_{i \in \omega} r_{i}\right)=\mathbb{R}^{n} \in W,
$$


then $t$ satisfies $\neg a \wedge[\mathrm{tpp}] \neg a \wedge[\mathrm{ntpp}] \neg a$ since, clearly, $t$ is not related via eq, tpp, and ntpp to any of the $r_{i}$. To show that Formula (6.2) holds, it thus suffices to prove that, for all $s \in W$ such that $s \neq t, \mathfrak{M}, s \models \neg[\mathrm{tppi}]\langle$ po $\rangle a$. Hence fix an $s \in W$ and assume that $s \neq t$. Since it is a region, $s$ is non-empty and regular closed. Therefore, we find a hyper-rectangle $h \in \mathbb{R}_{\text {rect }}^{n}$ contained in $s$. By expanding $h$ until we hit a point $x \in s-\mathbb{I} s$, we obtain an $h^{\prime} \in \mathbb{R}_{\text {rect }}^{n}$ such that $h \subseteq h^{\prime}$ and $h^{\prime}$ is a tangential proper part of $s$. Now fix an $x \in h^{\prime} \cap(s-\mathbb{I} s)$. Then, by the construction of the sequence $r_{1}, r_{2}, \ldots$, we can find a hyper-rectangle $h^{\prime \prime} \subseteq h^{\prime}$ which contains $x$ but is not in the relation po with any $r_{i}$. In conclusion, $\mathfrak{M}, h^{\prime \prime} \models[\mathrm{po}] \neg a$ and, therefore, $\mathfrak{M}, s \models\langle$ tppi $\rangle[$ po $] \neg a$.

Note that any region structure $\mathfrak{R}\left(\mathfrak{T}, \mathfrak{T}_{\text {reg }}\right)$, in particular the structures $\mathfrak{R}\left(\mathbb{R}^{n}, \mathbb{R}_{\text {reg }}^{n}\right)$, are closed under infinite unions. This applies as well to $\mathfrak{R}\left(\mathbb{R}^{n}, \mathbb{R}_{\text {conv }}^{n}\right)$. Since $\mathbb{R}_{\text {rect }}^{n} \subseteq \mathbb{R}_{\text {conv }}^{n} \subseteq$ $\mathbb{R}_{\text {reg }}^{n}$, Lemmas 17 and 18 immediately yield Theorem 15 .

It is worth noting that there are a number of interesting region structures to which this proof method does not apply. Interesting examples are the region structure of hyperrectangles in $\mathbb{R}^{n}, n \geq 2$, the region structure based on simply connected regions in $\mathbb{R}^{2}$ SS01, and the structure of polygons in $\mathbb{R}^{2}$ PS98. Since these spaces are not closed under infinite unions, the above proof does not show the non-axiomatizability of the induced logics. We believe, however, that slight modifications of the proof introduced here can be used to prove their $\Pi_{1}^{1}$-hardness as well.

\section{Finite Region Structures}

As discussed in Section 4 it can be useful to only admit models with a finite (but unbounded) number of regions. In this case, we can again establish a quite general undecidability result. Moreover, undecidability of a logic $L_{\mathrm{RCC} 8}^{\mathrm{fin}}(\mathcal{S})$ implies that it is not recursively enumerable if $\mathcal{S}$ is first-order definable. We start with proving undecidability.

Theorem 19. If $\mathfrak{R}\left(\mathbb{R}^{n}, \mathbb{R}_{\text {rect }}^{n}\right) \subseteq \mathcal{S} \subseteq \mathcal{R S}$ for some $n \geq 1$, then $L_{\mathrm{RCC} 8}^{\text {fin }}(\mathcal{S})$ is undecidable.

We obtain the following corollary.

Corollary 20. The following logics are undecidable for $n \geq 1: L_{\mathrm{RCC} 8}^{\mathrm{fin}}(\mathcal{R S}), L_{\mathrm{RCC} 8}^{\mathrm{fin}}(\mathcal{T O P})$, $L_{\mathrm{RCC} 8}^{\text {fin }}\left(\mathbb{R}^{n}, \mathbb{R}_{\text {reg }}^{n}\right), L_{\mathrm{RCC} 8}^{\text {fin }}\left(\mathbb{R}^{n}, \mathbb{R}_{\text {conv }}^{n}\right)$, and $L_{\mathrm{RCC} 8}^{\text {fin }}\left(\mathbb{R}^{n}, \mathbb{R}_{\text {rect }}^{n}\right)$.

To prove this result, we reduce yet another variant of the domino problem. For $k \in \mathbb{N}$, the $k$-triangle is the set $\{(i, j) \mid i+j \leq k\} \subseteq \mathbb{N}^{2}$. The task of the new domino problem is, given a domino system $\mathcal{D}=(T, H, V)$, to determine whether $\mathcal{D}$ tiles an arbitrary $k$-triangle, $k \in \mathbb{N}$, such that the position $(0,0)$ is occupied with a distinguished tile $s_{0} \in T$, and some position is occupied with a distinguished tile $f_{0} \in T$. It is shown in Appendix $\mathbf{D}$ that the existence of such a tiling is undecidable.

Given a domino system $\mathcal{D}$, the reduction formula $\varphi_{\mathcal{D}}$ is defined as

$$
a \wedge b \wedge \text { wall } \wedge \text { floor } \wedge s_{0} \wedge[\text { ntppi }] \neg a \wedge \square_{u} \chi \wedge\left(f_{0} \vee\langle\text { ntpp }\rangle\left(a \wedge b \wedge f_{0}\right)\right),
$$

where $\chi$ is the conjunction of the Formulas (5.1), (5.3) to (5.5), and (5.7) to (5.17) of Section [5] and the following formulas:

- The first tile that has no tile to the right is on the floor:

$$
\left(a \wedge b \wedge \neg \diamond^{R} \top \wedge\left[\text { ntppi] }\left((a \wedge b) \rightarrow \diamond^{R} \top\right)\right) \rightarrow\right. \text { floor }
$$


- If a tile has no tile to the right, then the next tile (if existent) also has no tile to the right:

$$
\left(a \wedge b \wedge \neg \diamond^{R} \top\right) \rightarrow\left(\neg \diamond^{+} \top \vee \diamond^{+} \neg \diamond^{R} \top\right)
$$

- The last tile is on the wall and we have no stacked orderings:

$$
\left(a \wedge b \wedge \neg \diamond^{+} \top\right) \rightarrow(\text { wall } \wedge[\mathrm{ntpp}] \neg(a \wedge b))
$$

The proof of the following lemma is now a variation of the proofs of Lemma 8 and Lemma 10. Details are left to the reader.

Lemma 21. Let $\mathcal{D}$ be a domino system. Then:

(i) if the formula $\varphi_{\mathcal{D}}$ is satisfiable in a finite region model, then $\mathcal{D}$ tiles a $k$-triangle for some $k \geq 1$;

(ii) if $\mathcal{D}$ tiles a $k$-triangle for some $k \geq 1$, then $\varphi_{\mathcal{D}}$ is satisfiable in a region model based on a finite substructure of $\mathfrak{R}\left(\mathbb{R}^{n}, \mathbb{R}_{\text {rect }}^{n}\right)$, for each $n \geq 1$.

Obviously, Theorem 19 is an immediate consequence of Lemma 21.

Since $\mathcal{R S}$ is first-order definable, we can enumerate all finite region models and also all formulas satisfiable in finite region models. Similarly, the proof of Theorem 14 shows that the class of at most countable substructures of $\mathfrak{R}\left(\mathbb{R}^{n}, \mathbb{R}_{\text {rect }}^{n}\right)$ is first-order definable (relative to the class of all at most countable structures), for $n \geq 1$. Thus, the complements of $L_{\mathrm{RCC} 8}^{\text {fin }}(\mathcal{R S})$ and $L_{\mathrm{RCC} 8}^{\mathrm{fin}}\left(\mathbb{R}^{n}, \mathbb{R}_{\text {rect }}^{n}\right)$ are recursively enumerable and Theorem [19] and Theorem 4 give us the following:

Corollary 22. The following logics are not r.e., for each $n \geq 1: L_{\mathrm{RCC} 8}^{\mathrm{fin}}(\mathcal{R S}), L_{\mathrm{RCC} 8}^{\mathrm{fin}}(\mathcal{T O P})$, $L_{\mathrm{RCC} 8}^{\text {fin }}\left(\mathbb{R}^{n}, \mathbb{R}_{\text {reg }}^{n}\right)$, and $L_{\mathrm{RCC} 8}^{\text {fin }}\left(\mathbb{R}^{n}, \mathbb{R}_{\text {rect }}^{n}\right)$.

We leave it as an open problem whether the logics $L_{\mathrm{RCC} 8}^{\text {fin }}\left(\mathbb{R}^{n}, \mathbb{R}_{\text {conv }}^{n}\right), n \geq 2$, are recursively enumerable.

\section{The RCC5 Set of Relations}

When selecting a set of relations between regions in topological spaces, the eight Egenhofer-Franzosa relations appear to be the most popular choice in the spatial reasoning community. However, it is not the only choice possible. For example, a refinement of RCC8 into 23 relations has been proposed and RCC5, a coarsening into five relations, is also rather popular GPP95, DWM01, Ben94, CH01. Since we have shown that modal logics based on the Egenhofer-Franzosa relations are undecidable and often even $\Pi_{1}^{1}$-complete, a natural next step for improving the computational behaviour is to consider modal logics based on a coarser set of relations. In this section, we define and investigate modal logics based on the RCC5 set of relations. It turns our that often reasoning is still undecidable, although different proof methods have to be used that yield less general theorems. For example, the recursive enumerability of modal logics determined by full concrete RCC5 region structures is left as an open problem.

The RCC5 set of relations is obtained from RCC8 by keeping the relations eq and po, but coarsening (1) the tpp and ntpp relations into a new "proper-part of" relation pp; (2) the tppi and ntppi relations into a new "has proper-part" relation ppi; and (3) the dc and ec relations into a new disjointness relation dr. Thus, a concrete $\mathrm{RCC5}$-structure $\mathfrak{R}^{5}\left(\mathfrak{T}, U_{\mathfrak{T}}\right)$ induced by a topological space $\mathfrak{T}$ and a set of regions $U_{\mathfrak{T}} \subseteq \mathfrak{T}_{\text {reg }}$ is the tuple $\left\langle U_{\mathfrak{T}}, \mathrm{eq}^{\mathfrak{R}}, \mathrm{po}^{\mathfrak{R}}, \mathrm{dr}^{\mathfrak{R}}, \mathrm{pp}^{\mathfrak{R}}, \mathrm{pp}^{\mathfrak{R}}\right\rangle$ where eq and po are interpreted as before and 


\begin{tabular}{|c||c|c|c|c|}
\hline$\circ$ & $\mathrm{dr}$ & po & pp & ppi \\
\hline \hline $\mathrm{dr}$ & $*$ & $\mathrm{dr}, \mathrm{po}, \mathrm{pp}$ & $\mathrm{dr}, \mathrm{po}, \mathrm{pp}$ & $\mathrm{dr}$ \\
\hline po & $\mathrm{dr}, \mathrm{po}, \mathrm{ppi}$, & $*$ & $\mathrm{po}, \mathrm{pp}$ & $\mathrm{dr}, \mathrm{po}, \mathrm{ppi}$ \\
\hline $\mathrm{pp}$ & $\mathrm{dr}$ & $\mathrm{dr}, \mathrm{po}, \mathrm{pp}$ & $\mathrm{pp}$ & $*$ \\
\hline ppi & $\mathrm{dr}, \mathrm{po}, \mathrm{ppi}$, & po,pp & eq,po,pp,ppi & ppi \\
\hline
\end{tabular}

Figure 6: The RCC5 composition table.

- $\mathrm{dr}^{\Re}=\mathrm{dc}^{\Re} \cup \mathrm{ec} \mathfrak{R}$

- $\mathrm{pp}^{\Re}=\mathrm{tpp}^{\Re} \cup \mathrm{nttp}^{\Re}$

- ppi $^{\Re}=$ tppi $^{\Re} \cup$ nttpi $i^{\Re}$.

It is interesting to note that the RCC5 relations can be defined without appealing to the topological notions of interior and closure. Hence, modal logics based on RCC5 may also be viewed as modal logics determined by the following relations between sets: 'having nonempty intersection', 'being disjoint', and 'is a subset of'. They are thus related to the logics considered in Vak95.

Similarly to concrete region structures induced by the eight Egenhofer-Franzosa relations, the class of concrete RCC5-structures can be characterized by first-order sentences. Denote by $\mathcal{R S}^{5}$ the class of all general RCC5-structures

$$
\left\langle W, \mathrm{dr}^{\Re}, \mathrm{eq}^{\mathfrak{R}}, \mathrm{pp}^{\mathfrak{R}}, \mathrm{ppi}^{\mathfrak{R}}, \mathrm{po}^{\mathfrak{R}}\right\rangle
$$

where $W$ is non-empty and the $r^{\Re}$ are mutually exclusive and jointly exhaustive binary relations on $W$ such that (1) eq is interpreted as the identity relation on $W,(2)$ po $^{\Re}$ and $\mathrm{dr}^{\mathfrak{R}}$ are symmetric, (3) $\mathrm{pp}^{\mathfrak{R}}$ is the inverse of $\mathrm{ppi}^{\mathfrak{R}}$ and (4) the rules of the RCC5-composition table (Figure 6) are valid.

The following representation theorem is proved by first establishing Point (ii) for finite RCC5-structures and then applying the same technique as in the proof of Theorem 1

\section{Theorem 23.}

(i) Every concrete RCC5-structure is a ageneral RCC5-structure;

(ii) every general RCC5-structure is isomorphic to a concrete RCC5-structure.

(iii) for every $n>0$, every countable general RCC5-structure is isomorphic to a concrete RCC5-structure of the form $\mathfrak{R}^{5}\left(\mathbb{R}^{n}, U_{\mathbb{R}^{n}}\right)$ (with $U_{\mathbb{R}^{n}} \subseteq \mathbb{R}_{\text {reg }}^{n}$ ).

As in the RCC8 case, we only distinguish between concrete and general RCC5-structures if necessary. RCC5-models are defined in the obvious way by extending RCC5-structures with a valuation function.

The modal language $\mathcal{L}_{\mathrm{RCC} 5}$ for reasoning about RCC5-structures extends propositional logic with unary modal operators [dr], [eq], etc. (one for each RCC5 relation). A number of results from our investigation of $\mathcal{L}_{\mathrm{RCC}}$ have obvious analogues for $\mathcal{L}_{\mathrm{RCC} 5}$.

The results established in Section 3 have counterparts in the RCC5 case: RCC5 constraint networks can be translated into $\mathcal{L}_{\mathrm{RCC}}$ in a straightforward way by defining nominals. Moreover, $\mathcal{L}_{\mathrm{RCC} 5}$ has the same expressive power as the two-variable fragment of $\mathcal{F} \mathcal{L}_{\mathrm{RCC} 5}^{m}$, i.e. the first-order language with the five binary RCC5-relation symbols and infinitely many unary predicates. Finally, the two-variable fragment of $\mathcal{F} \mathcal{L}_{\mathrm{RCC} 5}^{m}$ is exponentially more succinct on the class of structures $\mathcal{R S}^{5}$ than $\mathcal{L}_{\mathrm{RCC} 5}$. The proofs are analogous to those from Section 3 and Appendix B] 
Analogous to the RCC8 case, we define logics of full RCC5-structures, substructure variants, and finite substructure variants: given a class $\mathcal{S}$ of RCC5-structures, we denote with $L_{\mathrm{RCC} 5}(\mathcal{S})$ the set of $\mathcal{L}_{\mathrm{RCC} 5}$-formulas which are valid in all members of $\mathcal{S}$; with $L_{\mathrm{RCC} 5}^{\mathrm{S}}(\mathcal{S})$ the set of $\mathcal{L}_{\mathrm{RCC} 5}$-formulas which are valid in all substructures of members of $\mathcal{S}$; and with $L_{\mathrm{RCC} 5}^{\text {fin }}(\mathcal{S})$ the set of $\mathcal{L}_{\mathrm{RCC} 5}$-formulas which are valid in all finite substructures of members of $\mathcal{S}$. For brevity, we refrain from developing formulas that separate the different logics obtained by applying $\mathcal{L}_{\mathrm{RCC}}$ to different classes of RCC5-structures. Instead, we only note that there is an obvious analogue of Theorem 4 .

Theorem 24. For $n>0$, we have

(1) $L_{\mathrm{RCC} 5}(\mathcal{R S})=L_{\mathrm{RCC} 5}^{\mathrm{S}}(\mathcal{T O P})=L_{\mathrm{RCC} 5}^{\mathrm{S}}\left(\mathbb{R}^{n}, \mathbb{R}_{\mathrm{reg}}^{n}\right)$;

(2) $L_{\mathrm{RCC} 5}^{\text {fin }}(\mathcal{R S})=L_{\mathrm{RCC} 5}^{\text {fin }}(\mathcal{T O} \mathcal{P})=L_{\mathrm{RCC} 5}^{\text {fin }}\left(\mathbb{R}^{n}, \mathbb{R}_{\text {reg }}^{n}\right)$.

We now investigate the computational properties of logics based on $\mathcal{L}_{\mathrm{RCC} 5}$. Analogously to the RCC8 case, many natural logics are undecidable. Still, our RCC5 undecidability result is considerably less powerful than the one for RCC8. Intuitively, we have to restrict ourselves to RCC5-structures with the following property: for any set $S \subseteq W$ of cardinality two or three, there exists a unique smallest region $\operatorname{Sup}(S)$ that covers all regions from $S$. Formally, we define the class $\mathcal{R} \mathcal{S}^{\exists}$ of RCC5-structures $\left\langle W, \mathrm{dr}^{\Re}\right.$, eq $\left.{ }^{\Re}, \ldots\right\rangle$ satisfying the following condition: for every set $S \subseteq W$ of cardinality two or three, there exists a region $\operatorname{Sup}(S) \in W$ such that

- $s$ eq $\operatorname{Sup}(S)$ or $s$ pp $\operatorname{Sup}(S)$ for each $s \in S$;

- for every region $t \in W$ with $s$ pp $t$ for each $s \in S$, we have $\operatorname{Sup}(S)$ eq $t$ or $\operatorname{Sup}(S)$ pp $t$;

- for every region $t \in W$ with $t \mathrm{dr} s$ for each $s \in S$, we have $t \mathrm{dr} \operatorname{Sup}(S)$.

Region structures based on all non-empty regular closed sets in a topological space belong to $\mathcal{R S}^{\exists}$. This applies, in particular, to the structures $\mathfrak{R}^{5}\left(\mathbb{R}^{n}, \mathbb{R}_{\text {reg }}^{n}\right)$, for $n \geq 1$. However, their substructures usually do not belong to $\mathcal{R} \mathcal{S}^{\exists}$. For example, the structures $\mathfrak{R}^{5}\left(\mathbb{R}^{n}, \mathbb{R}_{\times}^{n}\right)$ with $\mathrm{x} \in\{$ conv, rect $\}$ and $n \geq 1$, are not in $\mathcal{R S}^{\exists}$. Our aim is to prove the following theorem:

Theorem 25. Suppose $\mathfrak{R}^{5}\left(\mathbb{R}^{n}, \mathbb{R}_{\text {reg }}^{n}\right) \in \mathcal{S} \subseteq \mathcal{R S}^{\exists}$, for some $n \geq 1$. Then $L_{\mathrm{RCC} 5}(\mathcal{S})$ is undecidable.

This clearly yields the following corollary:

Corollary 26. The following logics are undecidable, for each $n \geq 1: L_{\mathrm{RCC} 5}(\mathcal{T O P})$ and $L_{\mathrm{RCC5}}\left(\mathbb{R}^{n}, \mathbb{R}_{\text {reg }}^{n}\right)$.

The proof of Theorem [25] is by reduction of the satisfiability problem for the undecidable modal logic $\mathrm{S}^{3}$ to satifiability of $\mathcal{L}_{\mathrm{RCC} 5}$ formulas in $\mathcal{S}$. The original undecidability proof for $\mathrm{S}^{3}$ has been given by Maddux in an algebraic setting Mad80. For the reduction, we use the modal notation of [GKWZ03. More precisely, the language $\mathcal{L}_{3}$ is the extension of propositional logic by means of unary modal operators $\diamond_{1}, \diamond_{2}$ and $\diamond_{3}$. $\mathcal{L}_{3}$ is interpreted in $\mathrm{S} 5^{3}$-models

$$
\mathfrak{W}=\left\langle W_{1} \times W_{2} \times W_{3}, p_{1}^{\mathfrak{W}}, p_{2}^{\mathfrak{W}}, \ldots\right\rangle
$$

where the $W_{i}$ are non-empty sets and $p_{i}^{\mathfrak{W}} \subseteq W_{1} \times W_{2} \times W_{3}$. The truth-relation $\models$ between pairs $\left(\mathfrak{W},\left(w_{1}, w_{2}, w_{3}\right)\right)$ with $w_{i} \in W_{i}$, and $\mathcal{L}_{3}$-formulas $\varphi$ is defined inductively as follows:

- $\mathfrak{W},\left(w_{1}, w_{2}, w_{3}\right) \models p_{i}$ iff $\left(w_{1}, w_{2}, w_{3}\right) \in p_{i}^{\mathfrak{W}}$;

- $\mathfrak{W},\left(w_{1}, w_{2}, w_{3}\right) \models \neg \varphi$ iff $\mathfrak{W},\left(w_{1}, w_{2}, w_{3}\right) \not \models \varphi$;

- $\mathfrak{W},\left(w_{1}, w_{2}, w_{3}\right) \models \varphi_{1} \wedge \varphi_{2}$ iff $\mathfrak{W},\left(w_{1}, w_{2}, w_{3}\right) \models \varphi_{1}$ and $\mathfrak{W},\left(w_{1}, w_{2}, w_{3}\right) \models \varphi_{2}$;

- $\mathfrak{W},\left(w_{1}, w_{2}, w_{3}\right) \models \diamond_{1} \varphi$ iff there exists $w_{1}^{\prime} \in W_{1}$ such that $\mathfrak{W},\left(w_{1}^{\prime}, w_{2}, w_{3}\right) \models \varphi$; 
- $\mathfrak{W},\left(w_{1}, w_{2}, w_{3}\right) \models \diamond_{2} \varphi$ iff there exists $w_{2}^{\prime} \in W_{2}$ such that $\mathfrak{W},\left(w_{1}, w_{2}^{\prime}, w_{3}\right) \models \varphi$;

- $\mathfrak{W},\left(w_{1}, w_{2}, w_{3}\right) \models \diamond_{3} \varphi$ iff there exists $w_{3}^{\prime} \in W_{3}$ such that $\mathfrak{W},\left(w_{1}, w_{2}, w_{3}^{\prime}\right) \models \varphi$.

A formula $\varphi \in \mathcal{L}_{3}$ is called $\mathrm{S}^{3}{ }^{3}$-satisfiable if there exists an $\mathrm{S}^{3}{ }^{3}$-model $\mathfrak{W}$ and a triple $\left(w_{1}, w_{2}, w_{3}\right)$ such that $\mathfrak{W},\left(w_{1}, w_{2}, w_{3}\right) \models \varphi$.

Now for the reduction. The basic idea is to introduce three variables $a_{1}, a_{2}, a_{3}$ and then to represent each set $W_{i}$ of an $\mathrm{S}^{3}$-model by the set of pairwise disconnected regions

$$
\left\{r \in W \mid \mathfrak{M}, r \models a_{i}\right\} .
$$

The set $W_{1} \times W_{2} \times W_{3}$ is then represented by the set of regions

$$
\left\{\operatorname{Sup}\left(\left\{w_{1}, w_{2}, w_{3}\right\}\right) \mid \mathfrak{M}, w_{i} \models a_{i} \text { for } i \in\{1,2,3\}\right\} .
$$

The regions in this set will be marked with a variable $d$. To simulate the modal operators of $\mathbf{S}^{3}$, we will additionally refer to regions $\operatorname{Sup}\left(\left\{w_{i}, w_{j}\right\}\right)$ with $1 \leq i<j \leq 3$. Such regions are marked with the variable $d_{i, j}$.

The details of the reduction are as follows: with every $\mathrm{S}^{3}$-formula $\varphi$, we associate an $\mathcal{L}_{\mathrm{RCC} \text {-formula }}$

$$
\square_{u} \chi \wedge d \wedge \varphi^{\sharp}
$$

where $\varphi^{\sharp}$ is inductively defined below and $\chi$ is the conjunction of the following formulas:

(1) regions representing elements from $W_{1} \cup W_{2} \cup W_{3}$ are pairwise disconnected, each such region represents an element from $W_{i}$ for a unique $i$, and the sets $W_{i}$ are non-empty: for $i=1,2,3$, put

$$
\begin{gathered}
a_{i} \rightarrow \bigwedge_{j=1,2,3}\left([\mathrm{pp}] \neg a_{j} \wedge[\mathrm{ppi}] \neg a_{j} \wedge[\mathrm{po}] \neg a_{j}\right) \\
a_{1} \rightarrow \neg a_{2}, \quad a_{1} \rightarrow \neg a_{3}, \quad a_{2} \rightarrow \neg a_{3}, \\
\bigwedge_{i=1,2,3} \diamond_{u} a_{i}
\end{gathered}
$$

(2) the variable $d$ identifies regions representing elements of $W_{1} \times W_{2} \times W_{3}$ :

$$
d \leftrightarrow\left(\bigwedge_{i=1,2,3}\langle\text { ppi }\rangle a_{i}\right) \wedge \neg\langle\text { ppi }\rangle\left(\bigwedge_{i=1,2,3}\langle\text { ppi }\rangle a_{i}\right)
$$

(3) $d_{i, j}$ identifies regions representing elements of $W_{i} \times W_{j}$ : for $1 \leq i<j \leq 3$, put

$$
d_{i j} \leftrightarrow\left(\bigwedge_{k=i, j}\langle\text { ppi }\rangle a_{k}\right) \wedge \neg\langle\text { ppi }\rangle\left(\bigwedge_{k=i, j}\langle\text { ppi }\rangle a_{k}\right)
$$

Now, we define $\varphi^{\sharp}$ inductively by

$$
\begin{aligned}
p_{i}^{\sharp} & :=p_{i} \\
(\neg \varphi)^{\sharp} & :=d \wedge \neg \varphi^{\sharp} \\
(\varphi \wedge \psi)^{\sharp} & :=\varphi^{\sharp} \wedge \psi^{\sharp} \\
\left(\diamond_{1} \varphi\right)^{\sharp} & :=\langle\mathrm{ppi}\rangle\left(d_{23} \wedge\langle\mathrm{pp}\rangle\left(d \wedge \varphi^{\sharp}\right)\right) \\
\left(\diamond_{2} \varphi\right)^{\sharp} & :=\langle\mathrm{ppi}\rangle\left(d_{13} \wedge\langle\mathrm{pp}\rangle\left(d \wedge \varphi^{\sharp}\right)\right) \\
\left(\diamond_{3} \varphi\right)^{\sharp} & :=\langle\mathrm{ppi}\rangle\left(d_{12} \wedge\langle\mathrm{pp}\rangle\left(d \wedge \varphi^{\sharp}\right)\right)
\end{aligned}
$$

The following Lemma immediately yields Theorem 25. 
Lemma 27. Suppose $\mathfrak{R}\left(\mathbb{R}^{n}, \mathbb{R}_{\text {reg }}^{n}\right) \in \mathcal{S} \subseteq \mathcal{R} \mathcal{S}^{\exists}$, for some $n \geq 1$. Then an $\mathrm{S} 5^{3}$-formula $\varphi$ is satisfiable in an $\mathrm{S}^{3}$-model iff $\square_{u} \chi \wedge d \wedge \varphi^{\sharp}$ is satisfiable in $\mathcal{S}$.

Proof $(\Leftarrow)$ Suppose the region model

$$
\mathfrak{M}=\left\langle\mathfrak{R}, a_{1}^{\mathfrak{M}}, a_{2}^{\mathfrak{M}}, a_{3}^{\mathfrak{M}}, d^{\mathfrak{M}}, d_{12}^{\mathfrak{M}}, \ldots, p_{1}^{\mathfrak{M}}, \ldots\right\rangle
$$

satisfies $\square_{u} \chi \wedge d \wedge \varphi^{\sharp}$, where $\mathfrak{R}=\left\langle W, \mathrm{dr}^{\Re}, \mathrm{eq}^{\mathfrak{R}}, \ldots\right\rangle \in \mathcal{R} \mathcal{S}^{\exists}$. Define

$$
\mathfrak{W}=\left\langle W_{1} \times W_{2} \times W_{3}, p_{1}^{\mathfrak{W}}, p_{2}^{\mathfrak{W}}, \ldots\right\rangle
$$

by setting

- $W_{i}=a_{i}^{\mathfrak{M}}$, for $i=1,2,3$

- for all $\left(w_{1}, w_{2}, w_{3}\right) \in W_{1} \times W_{2} \times W_{3}$ and $i<\omega$, $\left(w_{1}, w_{2}, w_{3}\right) \in p_{i}^{\mathfrak{W}}$ iff $\operatorname{Sup}\left(\left\{w_{1}, w_{2}, w_{3}\right\}\right) \in p_{i}^{\mathfrak{M}}$.

By Formula (8.3), the $W_{i}$ are non-empty. Now, the function $f: W_{1} \times W_{2} \times W_{3} \rightarrow d^{\mathfrak{M}}$, defined by putting

is a well-defined bijection:

$$
f\left(w_{1}, w_{2}, w_{3}\right)=\operatorname{Sup}\left\{w_{1}, w_{2}, w_{3}\right\}
$$

- $f$ is well-defined (i.e., $\operatorname{Sup}\left\{w_{1}, w_{2}, w_{3}\right\} \in d^{\mathfrak{M}}$ ) by the properties of $\operatorname{Sup}(S)$ and by Formula (8.4);

- $f$ is injective since, by Formulas (8.1) and (8.2), we have $w_{1} \mathrm{dr} w_{2}$ for distinct $w_{1}, w_{2} \in W_{1} \cup W_{2} \cup W_{3}$. By the properties of $\operatorname{Sup}(S)$, we thus get $w$ dr $\operatorname{Sup}\left\{w_{1}, w_{2}, w_{3}\right\}$ for every $w \in W_{1} \cup W_{2} \cup W_{3}$ different from $w_{1}, w_{2}, w_{3}$;

- By Formula (8.4), $f$ is surjective.

Using Formula (8.5), one can show in the same way that $f_{i j}: W_{i} \times W_{j} \rightarrow d_{i j}^{\mathfrak{M}}, 1 \leq i<j \leq 3$, defined by

$$
f_{i j}\left(w_{i}, w_{j}\right)=\operatorname{Sup}\left\{w_{i}, w_{j}\right\}
$$

are well-defined bijections. Moreover, for all $\left(w_{1}, w_{2}, w_{3}\right) \in W_{1} \times W_{2} \times W_{3}$ and $u \in W_{i}$, $v \in W_{j}, 1 \leq i<j \leq 3$, we obtain $\operatorname{Sup}\{u, v\} \operatorname{pp} \operatorname{Sup}\left\{w_{1}, w_{2}, w_{3}\right\}$ iff $u=w_{i}$ and $v=w_{j}$.

Now it is straightforward to show by structural induction that, for all subformulas $\psi$ of $\varphi$ and all $\left(w_{1}, w_{2}, w_{3}\right) \in W_{1} \times W_{2} \times W_{3}$, we have

$$
\mathfrak{W},\left(w_{1}, w_{2}, w_{3}\right) \models \psi \text { iff } \mathfrak{M}, f\left(w_{1}, w_{2}, w_{2}\right) \models \psi^{\sharp} .
$$

Take $\left(w_{1}, w_{2}, w_{3}\right) \in W_{1} \times W_{2} \times W_{3}$ such that $f\left(w_{1}, w_{2}, w_{3}\right) \models \varphi^{\sharp}$. Then $\left(w_{1}, w_{2}, w_{3}\right) \models \varphi$. $(\Rightarrow)$ By the standard translation of $\mathrm{S}^{3}$ into first-order logic and the theorem of LöwenheimSkolem, every satisfiable $\mathrm{S}^{3}$ formula $\varphi$ is satisfiable in a countable model

$$
\mathfrak{W}=\left\langle W_{1} \times W_{2} \times W_{3}, p_{1}^{\mathfrak{W}}, p_{2}^{\mathfrak{W}}, \ldots\right\rangle .
$$

We may assume w.l.o.g. that the sets $W_{i}$ are mutually disjoint. Now let $n>0$ and define a model $\mathfrak{M}$ for $\square_{u} \chi \wedge d \wedge \varphi^{\sharp}$ based on the structure $\mathfrak{R}^{5}\left(\mathbb{R}^{n}, \mathbb{R}_{\text {reg }}^{n}\right)$ as follows. Let $f$ : $W_{1} \cup W_{2} \cup W_{3} \rightarrow \mathbb{R}_{\text {reg }}^{n}$ be an injective mapping such that $f(w) \operatorname{dr} f\left(w^{\prime}\right)$ if $w \neq w^{\prime}$, and set

- $a_{i}^{\mathfrak{M}}=\left\{f(w) \mid w \in W_{i}\right\}$, for $i=1,2,3$;

- $d^{\mathfrak{M}}=\left\{f\left(w_{1}\right) \cup f\left(w_{2}\right) \cup f\left(w_{3}\right) \mid\left(w_{1}, w_{2}, w_{3}\right) \in W_{1} \times W_{2} \times W_{3}\right\}$;

- $d_{i j}^{\mathfrak{M}}=\left\{f\left(w_{i}\right) \cup f\left(w_{j}\right) \mid\left(w_{i}, w_{j}\right) \in W_{i} \times W_{j}\right\}$, for $1 \leq i<j \leq 3$;

- $p_{i}^{\mathfrak{M}}=\left\{f\left(w_{1}\right) \cup f\left(w_{2}\right) \cup f\left(w_{3}\right) \mid\left(w_{1}, w_{2}, w_{3}\right) \models p_{i}\right\}$ for $i<\omega$. 
It is straightforward to prove that $\chi$ is true in every point of $\mathfrak{M}$. Moreover, one can easily prove by induction that, for every subformula $\psi$ of $\varphi$ and every $\left(w_{1}, w_{2}, w_{3}\right) \in W_{1} \times W_{2} \times W_{3}$, we have

$$
\mathfrak{W},\left(w_{1}, w_{2}, w_{3}\right) \models \psi \text { iff } \mathfrak{M}, f\left(w_{1}\right) \cup f\left(w_{2}\right) \cup f\left(w_{3}\right) \models \psi^{\sharp} .
$$

Since $\varphi$ is satisfied in $\mathfrak{W}$, we thus obtain that $\square_{u} \chi \wedge d \wedge \varphi^{\sharp}$ is satisfied in $\mathfrak{M}$.

The decidability of other RCC5 logics is left as an open problem. In particular, the decidability status of substructure logics and their finite companions is one of the most intriguing open problems suggested by the work presented in this paper.

Concering the recursive enumerability of logics based on $\mathcal{L}_{\mathrm{RCC} 5}$, we only note that a counterpart of Theorem 13 is easily obtained using an analogous proof:

Theorem 28. For $n>0, L_{\mathrm{RCC} 5}(\mathcal{R S})=L_{\mathrm{RCC} 5}^{\mathrm{S}}(\mathcal{T O P})=L_{\mathrm{RCC} 5}^{\mathrm{S}}\left(\mathbb{R}^{n}, \mathbb{R}_{\text {reg }}^{n}\right)$ are recursively enumerable.

As already noted, the recursive enumerability of RCC5 logics determined by full concrete RCC5-structures is left as an open problem.

\section{Conclusion}

We first compare our results with Halpern and Shoham results for interval temporal logic HS91. Although one might be tempted to conjecture that their undecidability proofs can be extended to logics of region spaces, a close inspection shows that the only spaces for which this might be possible are the logics of hyper-rectangles $L_{\mathrm{RCC} 8}\left(\mathbb{R}^{n}, \mathbb{R}_{\text {rect }}^{n}\right)$. An extension is not possible, however, for $L_{\mathrm{RCC} 8}(\mathcal{T O P})$ and $L_{\mathrm{RCC} 8}\left(\mathbb{R}^{n}, \mathbb{R}_{\text {reg }}^{n}\right)$, and not even for $L_{\mathrm{RCC} 8}^{\mathrm{S}}\left(\mathbb{R}^{n}, \mathbb{R}_{\text {rect }}^{n}\right)$. In fact, the proof technique developed in this paper is more powerful than that of [HS91: Theorems [5 [15] and 19] apply to logics induced by the region space $\mathfrak{R}\left(\mathbb{R}, \mathbb{R}_{\text {conv }}\right)$, which is clearly an interval structure. ${ }^{5}$ Interestingly, on this interval structure our results are stronger than those of Halpern and Shoham in two respects: first, we only need the RCC8 relations, which can be viewed as a "coarsening" of the Allen interval relations used by Halpern and Shoham. Second and more interestingly, by Theorem 5 we have also proved undecidability of the substructure logic $L_{\mathrm{RCC} 8}^{\mathrm{S}}\left(\mathbb{R}, \mathbb{R}_{\text {conv }}\right)$, which is a natural but much weaker variant of the full (interval temporal) logic $L_{\mathrm{RCC} 8}\left(\mathbb{R}, \mathbb{R}_{\text {conv }}\right.$ ), and not captured by Halpern and Shoham's undecidability proof.

Several open questions for future research remain. Similar to the temporal case, the main challenge is to exhibit a decidable and still useful variant of the logics proposed in this paper. Perhaps the most interesting candidate is $L_{\mathrm{RCC} 5}(\mathcal{R S})$, which coincides with the logics $L_{\mathrm{RCC} 5}^{\mathrm{S}}\left(\mathbb{R}^{n}, \mathbb{R}_{\text {reg }}^{n}\right)$, and to which the reduction exhibited in Section 8 does not apply. Other candidates could be obtained by modifying the set of relations, e.g. giving up some of them. It has, for example, been argued that dropping po still results in a useful formalism for applications in geographic information systems. An interesting step in this direction is [SS05], where a number of decidability and axiomatizability results are proved for modal logics over region structures with only one modal operator corresponding to certain inclusion relations between regions. Finally, it is an open problem whether $L_{\mathrm{RCC} 5}(\mathcal{R S})$ and

\footnotetext{
${ }^{5}$ Notice that Halpern and Shoham allow for intervals consisting of a single point while our intervals have to be regular closed sets and therefore non-singletons. However, as single point intervals are definable using the formula $[\mathrm{pp}] \perp$, all our negative results extend to interval structure with single point intervals.
} 
$L_{\mathrm{RCC5}}\left(\mathbb{R}^{n}, \mathbb{R}_{\text {reg }}^{n}\right)$ are recursively enumerable. Although we believe that they are r.e. (in contrast to their RCC8 counterparts), a proof is yet lacking.

\section{ACKNOWLEDGEMENT}

The authors wish to acknowledge helpful comments from two anonymous referees.

\section{REFERENCES}

[AF98] A. Artale and E. Franconi. A temporal description logic for reasoning about actions and plans. Journal of Artificial Intelligence Research (JAIR), 9:463-506, 1998.

[All83] J. F. Allen. Maintaining knowledge about temporal intervals. Communications of the ACM, 26(11), 1983.

[All84] J. F. Allen. Towards a general theory of action and time. Artificial Intelligence, 23(2):123-154, 1984.

[AvB02] M. Aiello and J. van Benthem. A modal walk through space. Journal of Applied Non-Classical logic, pages 319-363, 2002.

$\left[\mathrm{BCM}^{+} 03\right]$ F. Baader, D. Calvanese, D. McGuinness, D. Nardi, and P. F. Patel-Schneider, editors. The Description Logic Handbook: Theory, Implementation, and Applications. Cambridge University Press, 2003.

[Ben94] B. Bennett. Spatial reasoning with propositional logics. In J. Doyle, E. Sandewall, and P. Torasso, editors, Principles of Knowledge Representation and Reasoning: Proceedings of the 4th International Conference (KR94), San Francisco, CA., 1994. Morgan Kaufmann.

[Ben96] B. Bennett. Modal logics for qualitative spatial reasoning. Bulletin of the Interest Group in Pure and Applied Logic (IGPL), 4(1):23-45, 1996.

[Ben98] B. Bennett. Determining consistency of topological relations. Constraints, 3(2-3):213-225, June 1998.

[Bur80] J. Burgess. Decidability of branching time. Studia logica, 39:203-218, 1980.

[CCR93] Z. Cui, A. G. Cohn, and D. A. Randell. Qualitative and topological relationships in spatial databases. In D. Abel and B. C. Ooi, editors, Third International Symposium on Large Spatial Databases, SSD '93, volume 692 of Lecture Notes in Computer Science, pages 293-315. Springer, 1993.

[CH01] A. G. Cohn and S. Hazarika. Qualitative spatial representation and reasoning: An overview. Fundamenta Informaticae, 46:2-31, 2001.

[Cla85] B. Clarke. Individuals and points. Notre Dame Journal of Formal Logic, 26:61-75, 1985.

[Coh93] A. G. Cohn. Modal and non modal qualitative spatial logics. In F. D. Anger, H. M. Guesgen, and J. van Benthem, editors, Proceedings of the Workshop on Spatial and Temporal Reasoning, Chambéry, 1993. IJCAI-93.

[dR92] M. de Rijke. The modal logic of inequality. Journal of Symbolic Logic, 57:566-584, 1992.

[DW05] I. Düntsch and M. Winter. A representation theorem for Boolean contact algebras. Theoretical Computer Science (B), 347:498-512, 2005.

[DWM01] I. Düntsch, H. Wang, and S. McCloskey. A relation algebraic approach to the Region Connection Calculus. Theoretical Computer Science, 255:63-83, 2001.

[EF91] M. Egenhofer and R. Franzosa. Point-set topological spatial relations. International Journal of Geographical Information Systems, 5 (2):161-174, 1991.

[Ege94] M. Egenhofer. Deriving the composition of binary topological relations. Journal of Visual Languages and Computing, 5 (2):133-149, 1994.

[Eme90] E. A. Emerson. Temporal and modal logic. In J. van Leeuwen, editor, Handbook of Theoretical Computer Science, volume B, pages 995-1072. Elsevier and MIT Press, 1990.

[End72] H. Enderton. A Mathematical Introduction to Logic. Academic Press, 1972.

[EVW02] K. Etessami, M. Y. Vardi, and T. Wilke. First-order logic with two variables and unary temporal logic. Information and Computation, 179(2):279-295, 2002.

[Gab81a] D. Gabbay. An irreflexivity lemma with applications to axiomatizations of conditions on tense frames. In U. Mönnich, editor, Aspects of Philosophical logic, pages 67-89. Reidel, 1981. 
[Gab81b] D.M. Gabbay. Expressive functional completeness in tense logic. In U. Mönnich, editor, Aspects of Philosophical Logic, pages 91-117. Reidel, Dordrecht, 1981.

[Gal87] A. Galton. The logic of occurence. In A. Galton, editor, Temporal Logics and their Applications, pages 169-196. Academic Press, 1987.

[GHR94] D. M. Gabbay, I. M. Hodkinson, and M. A. Reynolds. Temporal Logic: Mathematical Foundations and Computational Aspects, Volume 1. Oxford University Press, Logic Guides 28, 1994.

[GKWZ03] D. Gabbay, A. Kurucz, F. Wolter, and M. Zakharyaschev. Many-dimensional Modal Logics: Theory and Applications. Elsevier, 2003.

[GP92] V. Goranko and S. Passy. Using the universal modality: gains and questions. Journal of Logic and Computation, 2:2-30, 1992.

[GPP95] M. Grigni, D. Papadias, and C. Papadimitriou. Topological inference. In Proceedings of the 14th International Joint Conference on Artificial Intelligence (IJCAI'95), pages 901-906. Morgan Kaufmann, 1995.

[GV93] G. Gargov and V.Goranko. Modal logic with names. Journal of Philosophical Logic, 22:607-636, 1993.

[GV01] V. Goranko and D. Vakarelov. Sahlqvist formulas in hybrid polyadic modal logics. Journal of Logic and Computation, 11:737-754, 2001.

[Har85] D. Harel. Recurring dominoes: Making the highly undecidable highly understandable. Annals of Discrete Mathematics, 24:51-72, 1985.

[HS91] J.Y. Halpern and Y. Shoham. A propositional modal logic of time intervals. Journal of the ACM, 38(4):935-962, 1991.

[KT44] J. Mc Kinsey and A. Tarski. The algebra of topology. Annals of Mathematics, pages 141-191, 1944.

[Lod00] K. Lodaya. Sharpening the undecidability of interval temporal logic. In Asian Computing Science Conference, pages 290-298, 2000.

[LSW01] C. Lutz, U. Sattler, and F. Wolter. Modal logic and the two-variable fragment. In Laurent Fribourg, editor, Computer Science Logic, number 2142 in Lecture Notes in Computer Science, pages 247-261. Springer-Verlag, 2001.

[Lut03] C. Lutz. Combining interval-based temporal reasoning with general TBoxes. Artificial Intelligence, 152(2):235-274, 2004.

[Mad80] R. Maddux. The equational theory of $c a_{3}$ is undecidable. Journal of Symbolic Logic, 45:311-315, 1980.

[MR99] M. Marx and M. Reynolds. Undecidability of compass logic. Journal of Logic and Computation, 9(6), 1999.

[NB95] B. Nebel and H. Bürckert. Reasoning about temporal relations: A maximal tractable subclass of Allen's interval algebra. Journal of the ACM, 42(1):43-66, 1995.

[Nut99] W. Nutt. On the translation of qualitative spatial reasoning problems into modal logics. In W. Burgard, T. Christaller, and A. B. Cremers, editors, KI-99: Advances in Artificial Intelligence, volume 1701 of LNAI, pages 113-124. Springer-Verlag, 1999.

[PH02] I. Pratt-Hartmann. A topological constraint language with component counting. Journal of Applied Non-Classical Logics, 12(3-4):441-467, 2002.

[PS98] I. Pratt and D. Schoop. A complete axiom system for polygonal mereotopology of the real plane. Journal of Philosophical Logic, 27:621-658, 1998.

[PSV99] C.H. Papadimitriou, D. Suciu, and V. Vianu. Topological queries in spatial databases. Journal of Computer and System Sciences, 58:29-53, 1999.

[Ras99] T. M. Rasmussen. Signed interval logic. In In Annual Conference of the European Association for Computer Science Logic (CSL'99), pages 157-171, 1999.

[RCC92] D.A. Randell, Z. Cui, and A.G. Cohn. A spatial logic based on regions and connection. In Proceedings of the 3rd International Conference on Knowledge Representation and Reasoning (KR'92), pages 165-176, San Mateo, 1992. Morgan Kaufmann.

[Ren02] J. Renz. A canonical model of the region connection calculus. Journal of Applied Non-classical Logic, 12:469-494, 2002.

[RN99] J. Renz and B. Nebel. On the complexity of qualitative spatial reasoning: A maximal tractable fragment of the region connection calculus. Artificial Intelligence, 108(1-2):69-123, 1999. 
[RZ01] M. Reynolds and M. Zakharyaschev. On the products of linear modal logics. Journal of Logic and Computation, 11:909-931, 2001.

[SS01] M. Schaefer and D. Stefankovic. Decidability of string graphs. In Proceedings of the thirty-third annual ACM symposium on Theory of Computing, pages 241-246. ACM Press, 2001.

[SS05] I. Shapirovsky and V. Shehtman. Modal logics of regions and Minkowski spacetime. Journal of Logic and Computation, 15:559-574, 2005.

[Ste00] J. G. Stell. Boolean connection algebras: A new approach to the region-connection calculus. Artificial Intelligence, 122:111-136, 2000.

[Vak95] D. Vakarelov. A modal logic for set relations. In Proceedings of the 10th International Congress of Logic, Methodology and Philosophy of Science, page 183, Florence, 1995.

[vB83] J. van Benthem. The Logic of Time. Reidel, 1983.

[Ven90] Y. Venema. Expressiveness and completeness of an interval tense logic. Notre Dame Journal of Formal Logic, 31(4):529-547, 1990.

[Ven92] Y. Venema. Multi-dimensional Modal Logic. PhD thesis, University of Amsterdam, 1992.

[VKV90] M. Vilain, H. Kautz, and P. Van Beek. Constraint propagation algorithms for temporal reasoning: A revised report. In D. S. Weld and J. de Kleer, editors, Readings in Qualitative Reasoning about Physical Systems, pages 373-381. Morgan Kaufmann, 1990.

[Wes01] M. Wessel. Obstacles on the way to qualitative spatial reasoning with description logics: Some undecidability results. In C. Goble, D. L. McGuinness, R. Möller, and P. F. Patel-Schneider, editors, Proceedings of the International Workshop in Description Logics 2001 (DL2001), number 49 in CEUR-WS (http://ceur-ws.org/), pages 96-105, 2001.

[Wol97] F. Wolter. The structure of lattices of subframe logics. Annals of Pure and Applied Logic, 86:47100, 1997. 


\section{Appendix A. Proof of Representation Theorem}

\section{Theorem 1 (Representation theorem).}

(i) Every concrete region structure is a general region structure;

(ii) every general region structure is isomorphic to a concrete region structure;

(iii) for every $n>0$, every countable general region structure is isomorphic to a concrete region structure of the form $\mathfrak{R}\left(\mathbb{R}^{n}, U_{\mathbb{R}^{n}}\right)$ (with $U_{\mathbb{R}^{n}} \subseteq \mathbb{R}_{\text {reg }}^{n}$ ).

The proof of this theorem refers to RCC8 constraint networks as introduced in Section 3 , with the only difference that, in the following, we also admit infinite such networks. For convenience, we repeat the definition here. An RCC8 constraint network is a set of constraints $(s \mathrm{r} r)$ with $s, r$ region variables and $\mathrm{r}$ an RCC8 relation. Such a network $N$ is satisfiable in a topological space $\mathfrak{T}$ with regions $U_{\mathfrak{T}}$ if there exists an assignment $\delta$ of regions in $U_{\mathfrak{T}}$ to region variables such that $(s \mathrm{r} r) \in N$ implies $\delta(s) \mathrm{r}^{\mathfrak{T}} \delta(r)$.

Proof (i) Is easily proved by verifying the conditions formulated for general region models. This includes verification of the composition table, c.f. CCR93.

(ii) is well-known for finite general region stuctures, see Ben98]. Thus, it remains to extend the result to infinite structures. We are going to prove this extension with the help of the compactness theorem for first-order logic. To this end, we reduce satisfiability of RCC8 constraint networks in topological spaces to satisfiability in certain relational structures. Fix a general region structure $\mathfrak{R}=\left\langle W, \mathrm{dc}^{\mathfrak{R}}, \mathrm{ec}^{\mathfrak{R}}, \ldots\right\rangle$ with $W$ infinite. An associated RCC8 constraint network, called the diagram of $\mathfrak{R}$ and denoted with $\operatorname{diag}(\mathfrak{R})$, is defined by

$$
\operatorname{diag}(\mathfrak{R})=\left\{\left(s_{w} \mathrm{r} s_{v}\right) \mid w, v \in W \text { and } \mathfrak{M} \models w \mathrm{r} v\right\},
$$

where the $s_{w}, w \in W$, are region variables. To prove (ii), it suffices to show that $\operatorname{diag}(\mathfrak{R})$ is satisfiable in some topological space $\mathfrak{T}$ with a set $U_{\mathfrak{T}}$ of non-empty regular closed regions: if this is the case, then

$$
\mathfrak{R}\left(\mathfrak{T},\left\{\delta\left(s_{w}\right) \mid w \in W\right\}\right)
$$

is a concrete region structure isomorphic to $\mathfrak{R}$, where $\delta$ is the assignment witnessing satisfaction of $\operatorname{diag}(\mathfrak{R})$ in $\left(\mathfrak{T}, U_{\mathfrak{T}}\right)$.

Recall that every partial order $(V, R)$ induces a topological space $\left(V, \mathbb{I}_{R}\right)$ by setting, for $X \subseteq V$,

$$
\mathbb{I}_{R} X=\{x \in V \mid \forall y(x R y \rightarrow y \in X)\}
$$

(and thus $\left.\mathbb{C}_{R} X=\{x \in V \mid \exists y(x R y \wedge y \in X)\}\right)$. We call $\left(V, \mathbb{I}_{R}\right)$ the topological space induced by $(V, R)$. Of particular interest for us are topological spaces induced by partial orders that are fork frames: a partial order $(V, R)$ is a fork frame if it is the disjoint union of forks, where a fork is a partial order $\left(\left\{x_{b}, x_{l}, x_{r}\right\}, S\right)$ such that $S$ is the reflexive closure of $\left\{\left(x_{b}, x_{l}\right),\left(x_{b}, x_{r}\right)\right\}$. For example, Figure 7 contains an example fork frame whose induced topological space satisfies the constraints $(r$ po $s),(s$ ec $t)$, and $(r$ dc $t)$ if $r, s$, and $t$ are interpreted as regular-closed sets as indicated. Denote by $\mathfrak{F}$ the class of all topological spaces based on fork frames. It is shown in Ben98, Ren02 that every finite constraint network which is satisfiable in a general region structure is satisfiable in a topological space $\mathfrak{T} \in \mathfrak{F}$ with regions $\mathfrak{T}_{\text {reg. }}$ As $\operatorname{diag}(\mathfrak{R})$ is trivially satisfiable in the general region structure $\mathfrak{R}$, every finite subset of $\operatorname{diag}(\mathfrak{R})$ is satisfiable in a topological space $\mathfrak{T} \in \mathfrak{F}$ with regions $\mathfrak{T}_{\text {reg }}$.

Next, we give a translation of subsets $N$ of the RCC8 constraint network $\operatorname{diag}(\mathfrak{R})$ to sets $\Gamma(N)$ of first-order sentences using a binary predicate $R$ for the partial order in fork frames, 


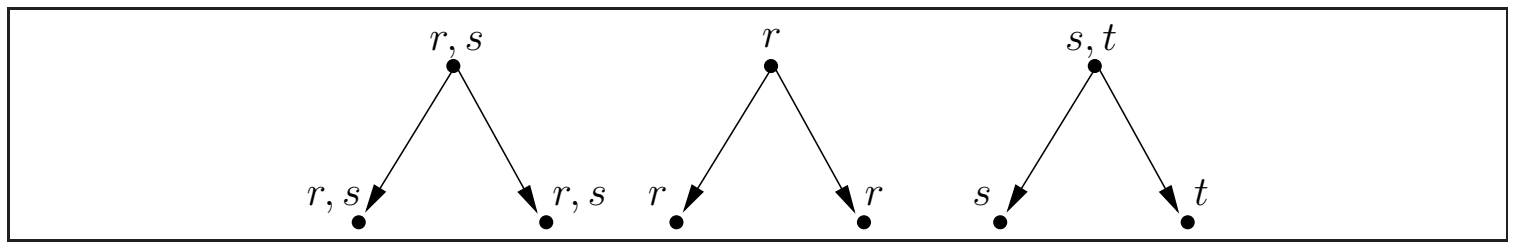

Figure 7: A fork frame satisfying $(r$ po $s),(s$ ec $t)$, and $(r$ dc $t)$.

and unary predicates $\left(P_{w}\right)_{w \in W}$ for regions. The translation is such that, for all $\mathfrak{T} \in \mathfrak{F}$ based on a fork frame $F=(V, S)$, the following conditions are equivalent:

- an assignment $\delta$ witnesses satisfaction of $N$ in $\mathfrak{T}$ with regions $\mathfrak{T}_{\text {reg; }}$;

- $\Gamma(N)$ is satisfied in the first-order structure $\mathfrak{M}$ with universe $V$ that is obtained by setting $R^{\mathfrak{M}}:=S$ and $P_{w}^{\mathfrak{M}}:=\delta\left(s_{w}\right)$ for all region variables $s_{w}$ in $N$.

The translation introduces one sentence for each constraint in $N$. We only treat the case $\left(s_{w}\right.$ ec $\left.s_{v}\right)$ :

$$
\exists x\left(P_{w}(x) \wedge P_{v}(x)\right) \wedge \neg \exists x\left(P_{w}(x) \wedge \forall y\left(x R y \rightarrow P_{v}(y)\right)\right) \wedge \neg \exists x\left(P_{v}(x) \wedge \forall y\left(x R y \rightarrow P_{w}(y)\right)\right) .
$$

The cases for other RCC8 relations are easily derived from their semantics and the definition of the topological spaces in $\mathfrak{F}$. Extend $\Gamma(N)$ to another set of first-order sentences $\Gamma^{*}(N)$ by adding the following:

- " $P_{w}$ is non-empty and regular closed", for all $w \in W$ :

$$
\exists x P_{w}(x) \wedge \forall x\left(P_{w}(x) \leftrightarrow \exists y\left(x R y \wedge \forall z\left(y R z \rightarrow P_{w}(z)\right)\right)\right) .
$$

- " $R$ is a disjoint union of forks" (details are left to the reader).

Clearly, $\Gamma^{*}(N)$ is satisfiable in an arbitrary first-order structure iff $\Gamma(N)$ is satisfied in a first-order structure $\mathfrak{M}$ obtained from a fork frame as described above iff $N$ is satisfiable in a topological space $\mathfrak{T} \in \mathfrak{F}$ with regions $\mathfrak{T}_{\text {reg }}$.

Thus, satisfiability of every finite subset of $\operatorname{diag}(\mathfrak{R})$ in a topological space $\mathfrak{T} \in \mathfrak{F}$ with regions $\mathfrak{T}_{\text {reg }}$ yields that every finite subset of $\Gamma^{*}(\operatorname{diag}(\mathfrak{R}))$ is satisfiable. By compactness of first-order logic, $\Gamma^{*}(\operatorname{diag}(\mathfrak{R}))$ is also satisfiable and thus $\operatorname{diag}(\mathfrak{R})$ is satisfiable in a topological space $\mathfrak{T} \in \mathfrak{F}$ with regions $\mathfrak{T}_{\text {reg }}$.

(iii) Suppose that $\mathfrak{R}=\left\langle W, \mathrm{dc}^{\mathfrak{R}}, \mathrm{ec}^{\Re}, \ldots\right\rangle$ is at most countable. From the encoding of constraint networks as sets of first-order sentences to be interpreted in fork frames and by Löwenheim-Skolem, we obtain that $\operatorname{diag}(\mathfrak{R})$ is satisfiable in a topological space $\mathfrak{T} \in \mathfrak{F}$ based on a fork frame $(V, S)$ with $V$ countable. Let $\delta$ be the assignment witnessing this satisfaction. To satisfy $\operatorname{diag}(\mathfrak{R})$ in $\mathbb{R}$ with regions $\mathbb{R}_{\text {reg }}$, assume that we have an enumeration $\left(\left\{x_{b}^{i}, x_{l}^{i}, x_{r}^{i}\right\}, S_{i}\right), i \in \mathbb{N}$, of the forks of $(V, S)$. To define an assignment $\delta^{\prime}$ in $\mathbb{R}_{\text {reg }}$, consider the sets

$$
W_{i}=\left\{w \in W \mid \delta\left(s_{w}\right) \supseteq\left\{x_{b}^{i}, x_{r}^{i}, x_{l}^{i}\right\}\right\}
$$

and take mappings $g_{i}$ from $W_{i}$ into the open interval $\left(\frac{1}{4}, \frac{1}{3}\right)$ such that

(1) $g_{i}(w) \leq g_{i}(v)$ if $\delta\left(s_{w}\right) \subseteq \delta\left(s_{v}\right)$;

(2) $g_{i}(w) \neq g_{i}(v)$ if $\delta\left(s_{w}\right) \neq \delta\left(s_{v}\right)$.

Such mappings exist because for each $S_{i}=\left\{\delta\left(s_{w}\right) \mid w \in W_{i}\right\}$ the partial order $\left(S_{i}, \subseteq\right)$ can be extended to a linear order which can then be embedded into the open interval $\left(\frac{1}{4}, \frac{1}{3}\right)$. 
Now set, for $w \in W$,

$\delta^{\prime}\left(s_{w}\right)=\bigcup_{i \in \mathbb{N}, x_{r}^{i} \in \delta(w), x_{l}^{i} \notin \delta(w)}\left[i, i+\frac{1}{4}\right] \cup \bigcup_{i \in \mathbb{N}, x_{l}^{i} \in \delta(w), x_{r}^{i} \notin \delta(w)}\left[i-\frac{1}{4}, i\right] \cup \bigcup_{i \in \mathbb{N}, x_{l}^{i}, x_{r}^{i} \in \delta(w)}\left[i-g_{i}(w), i+g_{i}(w)\right]$.

It is not hard to verify that each $\delta^{\prime}\left(s_{w}\right)$ is non-empty and regular closed since non-emptyness and regular closedness of $\delta\left(s_{w}\right)$ implies that $x_{b}^{i} \in \delta\left(s_{w}\right)$ iff $\left\{x_{l}^{i}, x_{r}^{i}\right\} \cap \delta\left(s_{w}\right) \neq \emptyset$. With the exception of the ntpp-case, we leave it to the reader to check that the assignment $\delta^{\prime}$ witnesses satisfaction of $\operatorname{diag}(\mathfrak{R})$ in $\mathbb{R}$ with regions $\mathbb{R}_{\text {reg. }}$. For ntpp, suppose that $\left(s_{w}\right.$ ntpp $\left.s_{v}\right) \in$ $\operatorname{diag}(\Re)$. Then $\delta\left(s_{w}\right)$ is in the relation ntpp to $\delta\left(s_{v}\right)$ in the topological space induced by $(V, S)$. We show that $\delta^{\prime}\left(s_{w}\right)$ is in the relation ntpp to $\delta^{\prime}\left(s_{v}\right)$ in $\mathbb{R}$. Clearly, by Condition 1 for the functions $g_{i}, \delta^{\prime}\left(s_{w}\right)$ is a subset of $\delta^{\prime}\left(s_{v}\right)$. To show that $\delta^{\prime}\left(s_{w}\right)$ is included in the interior of $\delta^{\prime}\left(s_{v}\right)$ we show that $\delta^{\prime}\left(s_{w}\right) \cap\left[i-\frac{1}{3}, i+\frac{1}{3}\right]$ is included in the interior of $\delta^{\prime}\left(s_{v}\right) \cap\left[i-\frac{1}{3}, i+\frac{1}{3}\right]$, for all $i \in \mathbb{N}$. Let $i \in \mathbb{N}$. We distinguish four cases.

- $\delta\left(s_{w}\right) \supseteq\left\{x_{r}^{i}, x_{l}^{i}\right\}$. Then $\delta\left(s_{v}\right) \supseteq\left\{x_{r}^{i}, x_{l}^{i}\right\}$ and therefore

$$
\delta^{\prime}\left(s_{u}\right) \cap\left[i-\frac{1}{3}, i+\frac{1}{3}\right]=\left[i-g_{i}(u), i+g_{i}(u)\right],
$$

for $u=w, v$. By Conditions 1 and 2 on the functions $g_{i},\left[i-g_{i}(w), i+g_{i}(w)\right]$ is included in the interior of $\left[i-g_{i}(v), i+g_{i}(v)\right]$.

- $x_{l}^{i} \in \delta\left(s_{w}\right)$ and $x_{r}^{i} \notin \delta\left(s_{w}\right)$. Then $\delta\left(s_{v}\right) \supseteq\left\{x_{r}^{i}, x_{l}^{i}\right\}$ (because otherwise $\delta\left(s_{w}\right)$ would not be included in the interior of $\left.\delta\left(s_{v}\right)\right)$. But then the claim follows from the fact that $\left[i-\frac{1}{4}, i\right]$ is in the interior of $\left[i-g_{i}(v), i+g_{i}(v)\right]$.

- $x_{r}^{i} \in \delta\left(s_{w}\right)$ and $x_{l}^{i} \notin \delta\left(s_{w}\right)$. Dual to the previous case.

- $\delta\left(s_{w}\right) \cap\left\{x_{r}^{i}, x_{r}^{i}\right\}=\emptyset$. Then $\delta^{\prime}\left(s_{w}\right) \cap\left[i-\frac{1}{3}, i+\frac{1}{3}\right]=\emptyset$ and the claim follows.

Assignments witnessing satisfaction of $\operatorname{diag}(\mathfrak{R})$ in $\mathbb{R}^{n}$ with regions $\mathbb{R}_{\text {reg }}^{n}, n>1$, can be constructed similarly using hyper-rectangles.

\section{Appendix B. Expressivity And Succinctness}

The proof of the following theorem is an adaptation of the proof in EVW02, and a minor variant of the proof in [LSW01] that is provided here for convenience. Throughout this section, we use $2 \mathcal{F} \mathcal{O}_{\mathrm{RCC} 8}^{m}$ to denote the two-variable fragment of $\mathcal{F} \mathcal{O}_{\mathrm{RCC} 8}^{m}$ and assume that its two variables are called $x$ and $y$.

Theorem 2, For every $2 \mathcal{F} \mathcal{O}_{\mathrm{RCC} 8}^{m}$-formula $\varphi(x)$ with free variable $x$, one can effectively construct a $\mathcal{L}_{\mathrm{RCC} 8}$-formula $\varphi^{*}$ of length at most exponential in the length of $\varphi(x)$ such that, for every region model $\mathfrak{M}$ and region $s$, we have $\mathfrak{M}, s \models \varphi^{*}$ iff $\mathfrak{M} \models \varphi[s]$.

Proof A $2 \mathcal{F} \mathcal{O}_{\mathrm{RCC}}^{m}$-formula $\xi$ is called a unary atom if it is of the form $\mathrm{r}(x, x), \mathrm{r}(y, y), p_{i}(x)$, or $p_{i}(y)$. It is called a binary atom if it is of the form $\mathrm{r}(x, y), \mathrm{r}(y, x), x=y$, or $y=x$. W.l.o.g. we assume that $2 \mathcal{F} \mathcal{O}_{\mathrm{RCC} 8}^{m}$-formulas are built using the operators $\exists, \wedge$, and $\neg$ only. We inductively define two mappings ${ }^{\sigma_{x}}$ and ${ }^{\sigma_{y}}$, the former taking each $2 \mathcal{F} \mathcal{O}_{\mathrm{RCC}}^{m}$-formula $\varphi(x)$ with free variable $x$ to the corresponding $\mathcal{L}_{\mathrm{RCC} 8}$-formula $\varphi^{\sigma_{x}}$, and the latter doing the same for $2 \mathcal{F O}_{\mathrm{RCC} 8}^{m}$-formulas $\varphi(y)$ with free variable $y$. We only give the details of ${ }^{\sigma_{x}}$ since $\sigma_{y}$ is defined analogously by switching the roles of $x$ and $y$ :

- If $\varphi(x)=p_{i}(x)$, then put $(\varphi(x))^{\sigma_{x}}=p_{i}$.

- If $\varphi(x)=\mathrm{r}(x, x)$, then put $(\varphi(x))^{\sigma_{x}}=\top$ if $\mathrm{r}=\mathrm{eq}$, and $(\varphi(x))^{\sigma_{x}}=\perp$ otherwise.

- If $\varphi(x)=\chi_{1} \wedge \chi_{2}$, then put $(\varphi(x))^{\sigma_{x}}=\chi_{1}^{\sigma_{x}} \wedge \chi_{2}^{\sigma_{x}}$. 
- If $\varphi(x)=\neg \chi$, then put $(\varphi(x))^{\sigma_{x}}=\neg\left(\chi^{\sigma_{x}}\right)$.

- If $\varphi(x)=\exists y \chi(x, y)$, then $\chi(x, y)$ can be written as

$$
\chi(x, y)=\gamma\left[\rho_{1}, \ldots, \rho_{r}, \gamma_{1}(x), \ldots, \gamma_{l}(x), \xi_{1}(y), \ldots, \xi_{s}(y)\right],
$$

i.e. as a Boolean combination $\gamma$ of $\rho_{i}, \gamma_{i}(x)$, and $\xi_{i}(y)$, where the $\rho_{i}$ are binary atoms, the $\gamma_{i}(x)$ are unary atoms or of the form $\exists y \gamma_{i}^{\prime}$, and the $\xi_{i}(y)$ are unary atoms or of the form $\exists x \xi_{i}^{\prime}$. We may assume w.l.o.g. that $x$ occurs free in $\varphi(x)$. Our first step is to move all formulas without a free variable $y$ out of the scope of $\exists$ : obviously, $\varphi(x)$ is equivalent to

$$
\bigvee_{\left\langle w_{1}, \ldots, w_{\ell}\right\rangle \in\{\top, \perp\}^{\ell}}\left(\bigwedge_{1 \leq i \leq \ell}\left(\gamma_{i} \leftrightarrow w_{i}\right) \wedge \exists y \gamma\left(\rho_{1}, \ldots, \rho_{r}, w_{1}, \ldots, w_{l}, \xi_{1}, \ldots, \xi_{s}\right)\right) .
$$

Now we "guess" a relation $\mathrm{r}$ that holds between $x$ and $y$, and then replace all binary atoms by either true or false according to the guess. For $r$ an RCC8 relation and $1 \leq i \leq r$, let

- $\rho_{i}^{r}=\top$ if $\rho_{i}=\mathrm{r}(x, y)$;

- $\rho_{i}^{r}=\top$ if $\rho_{i}=r(y, x)$ for $r \in\{\mathrm{dc}$, ec, po $\}$;

- $\rho_{i}^{\mathrm{r}}=\top$ if $\rho_{i}=\operatorname{tpp}(y, x)$ and $\mathrm{r}=\operatorname{tppi}$ or $\rho_{i}=\operatorname{ntpp}(y, x)$ and $\mathrm{r}=\mathrm{ntppi}$;

- $\rho_{i}^{\mathrm{r}}=\top$ if $\rho_{i}$ is $x=y$ and $\mathrm{r}=$ eq;

- $\rho_{i}^{r}=\perp$ otherwise.

Using this notiation, $(*)$ is equivalent to

$$
\begin{aligned}
\bigvee_{\left\langle w_{1}, \ldots, w_{\ell}\right\rangle \in\{\top, \perp\}^{\ell}}\left(\bigwedge_{1 \leq i \leq \ell}\left(\gamma_{i} \leftrightarrow w_{i}\right) \wedge\right. \\
\left.\bigvee_{\mathrm{r} \in \mathrm{RCC} 8} \exists y\left(\mathrm{r}(x, y) \wedge \gamma\left(\rho_{1}^{\mathrm{r}}, \ldots, \rho_{r}^{\mathrm{r}}, w_{1}, \ldots, w_{l}, \xi_{1}, \ldots, \xi_{s}\right)\right)\right) .
\end{aligned}
$$

Now compute, recursively, $\gamma_{i}^{\sigma_{x}}$ and $\xi_{i}^{\sigma_{y}}$, and define $\varphi(x)^{\sigma}$ as

$$
\begin{aligned}
& \bigvee_{\left\langle w_{1}, \ldots, w_{\ell}\right\rangle \in\{\top, \perp\}^{\ell}}\left(\bigwedge_{1 \leq i \leq \ell}\left(\gamma_{i}^{\sigma_{x}} \leftrightarrow w_{i}\right) \wedge\right. \\
& \left.\bigvee_{\mathrm{r} \in \mathrm{RCC} 8}\langle\mathrm{r}\rangle \gamma\left(\rho_{1}^{\mathrm{r}}, \ldots, \rho_{r}^{\mathrm{r}}, w_{1}, \ldots, w_{l}, \xi_{1}^{\sigma_{y}}, \ldots, \xi_{s}^{\sigma_{y}}\right)\right)
\end{aligned}
$$

Theorem 3. For $n \geq 1$, define a $\mathcal{F} \mathcal{O}_{\mathrm{RCC} 8}^{m}$ formula

$$
\varphi_{n}:=\forall x \forall y\left(\bigwedge_{i<n}\left(p_{i}(x) \leftrightarrow p_{i}(y)\right) \rightarrow\left(p_{n}(x) \leftrightarrow p_{n}(y)\right)\right)
$$

Then every $\mathcal{L}_{\mathrm{RCC}}$-formula $\psi_{n}$ that is equivalent to $\varphi_{n}$ on the class of all region structures $\mathcal{R S}$ has length $2^{\Omega(n)}$.

Proof Etessami et al. EVW02] show that, on $\omega$-words, every temporal logic formula equivalent to $\varphi_{n}$ is of length at least $2^{\Omega(n)}$, where temporal logic is assumed to have the operators "next", "previously", "always in the future" $\left(\square^{+} \varphi\right)$, and "always in the past" $\left(\square^{-} \varphi\right)$. Assume, to the contrary of what is to be shown, that there is an $n \geq 1$ and an $\mathcal{L}_{\mathrm{RCC}}$ formula $\psi$ such that $\psi$ is equivalent to $\varphi_{n}$ on the class of structures $\mathcal{R S}$ and the length of $\psi$ is smaller than $2^{\Omega(n)}$. Let $\mathfrak{R}=\left\langle W, \mathrm{dc}^{\Re}, \mathrm{ec}^{\Re}, \ldots\right\rangle \in \mathcal{R} \mathcal{S}$ be such that $W=\left\{s_{0}, s_{1}, s_{2}, \ldots\right\}$ and $s_{i} \operatorname{ntpp}^{\mathfrak{R}} s_{j}$ if $j>i$. Clearly, $\psi$ is equivalent to $\varphi_{n}$ on $\mathfrak{R}$. We construct a new formula $\psi^{*}$ by exhaustively performing the following rewritings on (subformulas of) $\psi:^{6}$

- $[r] \vartheta \rightsquigarrow \top$ if $r \notin\{n t p p, n t p p i\} ;$

- $[\mathrm{eq}] \vartheta \rightsquigarrow \vartheta$.

\footnotetext{
${ }^{6}$ Recall that $\langle r\rangle \vartheta$ is only an abbreviation.
} 
The formula $\psi^{*}$ is equivalent to $\psi$ (and thus to $\varphi_{n}$ ) on $\mathfrak{R}$, it only refers to the relations ntpp and ntppi, and it may only be shorter, but not longer than $\psi$. We may now convert $\psi^{*}$ into a temporal logic formula $\psi^{t}$ by substituting subformulas [ntpp] $\vartheta$ with $\square^{+} \vartheta$ and subformulas [ntppi] $\vartheta$ with $\square^{-} \vartheta$. It is not hard to see that $\psi^{t}$ is equivalent to $\varphi_{n}$ on $\omega$-words. Thus, we have derived a contradiction to the fact that there is no such temporal logic formula of length smaller than $2^{\Omega(n)}$.

\section{Appendix C. Recursive Enumerability of $L_{\mathrm{RCC} 8}^{\mathrm{S}}\left(\mathbb{R}^{n}, \mathbb{R}_{\text {rect }}^{n}\right)$}

Theorem 14, For $n \geq 1, L_{\mathrm{RCC} 8}^{\mathrm{S}}\left(\mathbb{R}^{n}, \mathbb{R}_{\text {rect }}^{n}\right)$ is recursively enumerable.

Proof We show this result for $n=2$. For $n=1$ and $n>2$, the proof is similar and left to the reader. Take the first-order language $\mathcal{F} \mathcal{L}_{4}$ with one binary relation symbol $<$, infinitely many 4 -ary relation symbols $P_{1}, P_{2}, \ldots$, and one extra 4 -ary relation symbol exists. Define a 4 -ary predicate $\operatorname{rect}\left(x_{1}, x_{2}, x_{3}, x_{4}\right)$ by setting

$$
\operatorname{rect}\left(x_{1}, x_{2}, x_{3}, x_{4}\right)=\left(x_{1}<x_{2}\right) \wedge\left(x_{3}<x_{4}\right) .
$$

Clearly, we can identify any vector $\vec{a}=\left(a_{1}, a_{2}, a_{3}, a_{4}\right) \in \mathbb{R}^{2}$ such that $\mathbb{R}=\operatorname{rect}(\vec{a})$ with the rectangle

$$
\left[a_{1}, a_{2}\right] \times\left[a_{3}, a_{4}\right] \in \mathbb{R}_{\text {rect }}^{2} .
$$

Moreover, it is easy (but tedious) to find, for every RCC8 relation $r$, a $\mathcal{F} \mathcal{L}_{4}$ formula $\varphi_{\mathrm{r}}\left(x_{1}, \ldots, x_{4}, y_{1}, \ldots y_{4}\right)$ such that, for any two rectangles $\left[a_{1}, a_{2}\right] \times\left[a_{3}, a_{4}\right]$ and $\left[b_{1}, b_{2}\right] \times\left[b_{3}, b_{4}\right]$, we have

$$
\left[a_{1}, a_{2}\right] \times\left[a_{3}, a_{4}\right] \mathrm{r}\left[b_{1}, b_{2}\right] \times\left[b_{3}, b_{4}\right] \text { iff } \mathbb{R}^{2} \models \varphi_{\mathrm{r}}(\vec{a}, \vec{b}) .
$$

The details of working out these formulas are left to the reader. Now fix variables $\vec{x}=$ $x_{1}, \ldots, x_{4}$ and $\vec{y}=y_{1}, \ldots, y_{4}$, and define a translation $s$ from $\mathcal{L}_{\mathrm{RCC} 8}$ into $\mathcal{F} \mathcal{L}_{4}$ by

$$
\begin{aligned}
p_{i}^{s} & =\operatorname{rect}(\vec{x}) \wedge \operatorname{exists}(\vec{x}) \wedge P_{i}(\vec{x}) \\
\left(\psi_{1} \wedge \psi_{2}\right)^{s} & =\psi_{1}^{s} \wedge \psi_{2}^{s} \\
(\neg \psi)^{s} & =\operatorname{rect}(\vec{x}) \wedge \operatorname{exists}(\vec{x}) \wedge \neg \psi^{s} \\
(\langle r\rangle \psi)^{s} & =\operatorname{rect}(\vec{x}) \wedge \operatorname{exists}(\vec{x}) \wedge \exists \vec{y}\left(\varphi_{\mathrm{r}}(\vec{x}, \vec{y}) \wedge \psi^{s}(\vec{y} / \vec{x})\right) .
\end{aligned}
$$

Claim. For every formula $\varphi \in \mathcal{L}_{\mathrm{RCC} 8}, \varphi$ is satisfiable in a substructure of $\mathfrak{R}\left(\mathbb{R}^{2}, \mathbb{R}_{\text {rect }}^{2}\right)$ iff $\varphi^{s}$ is satisfiable in a first-order model of the form $\mathfrak{Q}=\left(\mathbb{Q},<\right.$ exists $\left.^{\mathfrak{Q}}, P_{1}^{\mathfrak{Q}}, P_{2}^{\mathfrak{Q}}, \ldots\right)$.

$(\Rightarrow)$ Suppose $\varphi$ is satisfied in a region model $\mathfrak{M}$ based on a substructure of $\mathfrak{R}\left(\mathbb{R}^{2}, \mathbb{R}_{\text {rect }}^{2}\right)$. Then $\varphi^{s}$ is satisfiable in the first-order model

$$
\mathfrak{R}=\left(\mathbb{R},<, \text { exists }^{\Re}, P_{1}^{\Re}, P_{2}^{\Re}, \ldots\right)
$$

in which exists is interpreted as the set of all rectangles belonging to the domain of $\mathfrak{M}$ and the $P_{i}$ are interpreted as the set of rectangles in which $p_{i}$ is true in $\mathfrak{M}$. By LöwenheimSkolem, there exists a countably infinite elementary substructure of $\mathfrak{R}$ in which $\varphi^{s}$ is satisfied (see [End72]). Clearly, this structure is a dense linear order without endpoints. As every countable dense linear order without endpoints is isomorphic to $(\mathbb{Q},<)$, this structure is of the form required.

$(\Leftarrow)$ Suppose $\varphi^{s}$ is satisfiable in $\mathfrak{Q}=\left(\mathbb{Q},<\right.$, exists $\left.{ }^{\mathfrak{Q}}, P_{1}^{\mathfrak{Q}}, P_{2}^{\mathfrak{Q}}, \ldots\right)$. Define a region model $\mathfrak{M}$ based on a substructure of $\mathfrak{R}\left(\mathbb{R}^{2}, \mathbb{R}_{\text {rect }}^{2}\right)$ with domain $U$ and valuation $\mathfrak{V}$ as follows: let $U$ denote the set of rectangles of the form $\left[a_{1}, a_{2}\right] \times\left[a_{3}, a_{4}\right]$ such that $\mathfrak{Q} \models \operatorname{rect}(\vec{a}) \wedge \operatorname{exists}(\vec{a})$. 
Let $\mathfrak{V}\left(p_{i}\right)$ be the set of all rectangles $\vec{a}$ in $U$ such that $\mathfrak{Q} \models P_{i}(\vec{a})$. Then it is readily checked that $\mathfrak{M}$ satisfies $\varphi$.

This finishes the proof of the claim. Now set $\varphi^{t}=\forall \vec{x}\left(\operatorname{rect}(\vec{x}) \wedge \operatorname{exists}(\vec{x}) \rightarrow \varphi^{s}\right)$, for every $\varphi \in \mathcal{L}_{\mathrm{RCC} 8}$. Moreover, let $\Gamma$ be the conjunction of the usual first-order axioms for dense linear orders without endpoints (see e.g. End72]). It follows from the claim above that $\varphi$ is valid in all substructures of $\Re\left(\mathbb{R}^{2}, \mathbb{R}_{\text {rect }}^{2}\right)$ iff $\Gamma \rightarrow \varphi^{t}$ is a theorem of first-order logic. Thus, recursive enumerability of $L_{\mathrm{RCC} 8}^{\mathrm{S}}\left(\mathbb{R}^{2}, \mathbb{R}_{\text {rect }}^{2}\right)$ is obtained from recursive enumerability of first-order logic.

\section{Appendix D. The Domino Problem for $k$-Triangles}

Recall that, for $k \in \mathbb{N}$, the $k$-triangle is the set $\{(i, j) \mid i+j \leq k\} \subseteq \mathbb{N}^{2}$. We are going to prove the following undecidability result:

Theorem 29. Given a domino system $\mathcal{D}=(T, H, V)$, it is undecidable whether $\mathcal{D}$ tiles a $k$-triangle, $k \geq 1$, such that the position $(0,0)$ is occupied by a distinguished tile $s_{0} \in T$ and some position is occupied by a distinguished tile $f_{0} \in T$.

The proof is via a reduction of the halting problem for Turing machines with a single right-infinite tape that are started on the empty tape. The basic idea of the proof is to represent a run of the Turing machine as a sequence of columns of a $k$-triangle, where each column represents a configuration (with the left-most tape cell at the bottom of the column). Let $\mathfrak{A}$ be a single-tape right-infinite Turing machine with state space $Q$, initial state $q_{0}$, halt state $q_{f}$, tape alphabet $\Sigma(b \in \Sigma$ stands for blank), and transition relation $\Delta \subseteq Q \times \Sigma \times Q \times \Sigma \times\{L, R\}$. W.l.o.g., we assume that Turing machines have the following properties:

- the initial state $q_{0}$ is only used at the beginning of computations, but not later;

- the TM comes to a stop only if it reaches $q_{f}$;

- if the TM halts, its last step is to the right;

- if the TM halts, then it labels the halting position with a special symbol $\# \in \Sigma$ before;

- the blank symbol is never written.

It is easily checked that every TM can be modified to satisfy these requirements. The configurations of $\mathfrak{A}$ will be represented by finite words of one of the forms

(1) $x b^{m}$

(2) $a_{0} \cdots a_{k} x y a_{0}^{\prime} \cdots a_{\ell}^{\prime} b^{m}$,

(3) $a_{0} \cdots a_{k} y x a_{0}^{\prime} \cdots a_{\ell}^{\prime} b^{m}$

where

- $m>0$,

- all $a_{i}$ and $a_{i}^{\prime}$ are in $\Sigma$,

- $x \in A:=Q \times \Sigma \times\{L, R\}$ represents the active tape cell, its content, the current state, and the direction to which the TM has moved to reach the current position, and

- $y \in A^{\dagger}:=\left\{\langle q, \sigma, M\rangle^{\dagger} \mid\langle q, \sigma, M\rangle \in A\right\}$ represents the previously active tape cell, its current content, the current state, and the direction to which $\mathfrak{A}$ moved to reach the current position. 
Note that the only difference between elements of $A$ and elements of $A^{\dagger}$ is that the latter are marked with the symbol "†". Intuitively, the elements of $A$ describe the current head position while the elements of $A^{\dagger}$ describe the previous one. For technical reasons, the information whether the last step was to the left or to the right is stored twice in each column: both in the $x$ cell and in the $y$ cell. Configurations of Form 1 represent the initial configuration and thus do not comprise the description of a previous state.

Given a Turing machine $\mathfrak{A}$, we define a domino system $\mathcal{D}_{\mathfrak{A}}=\left(T, H, V, s_{0}, f_{0}\right)$ as follows:

- $T:=\Sigma \cup A \cup A^{\dagger} \cup\{\$\}$;

- $s_{0}:=\left\langle q_{0}, b, L\right\rangle$

- $f_{0}:=\left\langle q_{f}, \#, R\right\rangle$

- $H:=\{(\sigma, \sigma) \mid \sigma \in \Sigma\} \cup$

$$
\begin{aligned}
& \left\{\left(\langle q, \sigma, M\rangle,\left\langle q^{\prime}, \sigma^{\prime}, M^{\prime}\right\rangle^{\dagger}\right) \mid\left(q, \sigma, q^{\prime}, \sigma^{\prime}, M^{\prime}\right) \in \Delta, M \in\{L, R\}\right\} \cup \\
& \left\{(\sigma,\langle q, \sigma, M\rangle),\left(\langle q, \sigma, M\rangle^{\dagger},\left\langle q^{\prime}, \sigma, M^{\prime}\right\rangle\right) \mid \sigma \in \Sigma, q, q^{\prime} \in Q, M, M^{\prime} \in\{L, R\}\right\} \cup \\
& \left\{\left(\langle q, \sigma, M\rangle^{\dagger}, \sigma\right) \mid q \in Q, \sigma \in \Sigma, M \in\{L, R\}\right\} \cup \\
& \{(\langle q f, \#, R\rangle, \$),(\$, \$)\} \cup\{(\sigma, \$) \mid \sigma \in \Sigma\} \cup \\
& \left\{\left(\langle q, \sigma, M\rangle^{\dagger} \$\right) \mid q \in Q, M \in\{L, R\}\right\} \\
\bullet V:= & \left\{\left(\sigma, \sigma^{\prime}\right) \in \Sigma^{2} \mid \sigma=b \operatorname{implies} \sigma^{\prime}=b\right\} \cup \\
& \left\{\left(\sigma,\left\langle q, \sigma^{\prime}, L\right\rangle\right),\left(\left\langle q, \sigma^{\prime}, R\right\rangle, \sigma\right) \mid \sigma, \sigma^{\prime} \in \Sigma, q \in Q\right\} \cup \\
& \left\{\left(\left\langle q, \sigma^{\prime}, L\right\rangle^{\dagger}, \sigma\right),\left(\sigma,\left\langle q, \sigma^{\prime}, R\right\rangle^{\dagger}\right) \mid \sigma, \sigma^{\prime} \in \Sigma, q \in Q\right\} \cup \\
& \left\{\left(\langle q, \sigma, L\rangle,\left\langle q, \sigma^{\prime}, L\right\rangle^{\dagger}\right),\left(\left\langle q, \sigma^{\prime}, R\right\rangle^{\dagger},\langle q, \sigma, R\rangle\right) \mid \sigma, \sigma^{\prime} \in \Sigma, q \in Q\right\} \cup
\end{aligned}
$$

The tile " $\$$ " is used for padding purposes: assume that there exists a terminating computation of $\mathcal{A}$ on the empty tape. Then this computation induces in an obvious way the tiling of a finite rectangle such that $s_{0}$ is at position $(0,0), f_{0}$ occurs somewhere in the right-most column, and the height of the rectangle is bounded by the width $w$ of the rectangle. We may now perform a padding of the columns and rows in order to extend this rectangle to a $2 w$-triangle: for extending the height of columns, we may pad with the blank symbol " $b$ ", and for extending the width of rows, we may pad with the special symbol "\$". Since the existence of a tiling of a $k$-rectangle with $s_{0}$ at position $(0,0)$ and $f_{0}$ occurring somewhere induces a halting computation of $\mathcal{A}$ in a straightforward way, we obtain the following lemma.

Lemma 30. The Turing machine $\mathcal{A}$ halts on the empty tape iff the domino system $\mathcal{D}_{\mathfrak{A}}$ tiles a $k$-triangle, for some $k \geq 1$, such that position $(0,0)$ is occupied by the tile $s_{0}$ and some position is occupied by $f_{0}$.

Finally, Theorem 29 is an immediate consequence of Lemma 30.

This work is licensed under the Creative Commons Attribution-NoDerivs License. To view a copy of this license, visit http://creativecommons.org/licenses/by-nd/2.0/ or send a letter to Creative Commons, 559 Nathan Abbott Way, Stanford, California 94305, USA. 